\title{
OBSERVATIONS SUR LA BIOLOGIE DE BULINUS TRUNCATUS AU KHOUZISTAN (IRAN)
}

\author{
Par Jean GAUD, Fayedoum ARFAA et Ahmad ZEINI
}

SOMMAIRE

I. Introduction :

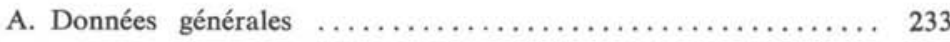

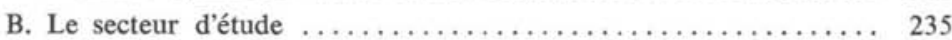

II. Aspects écologiques généraux :

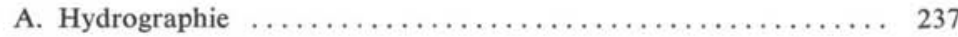

B. Limnologie .................................. 239

C. Rôle de l'homme dans l'écologie de B. truncatus .......... 243

III. Répartition de B. truncatus :

A. Modes et valeur de la prospection effectuée ............ 247

B. Distribution de B. truncatus dans le secteur étudié .......... 253

C. Distribution des bulins et \& physiologie » de l'irrigation ..... 257

IV. Evolution des populations de B. truncatus :

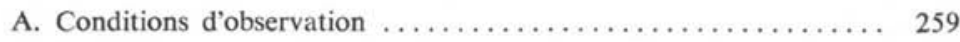

B. Rapidité de développement des populations de bulins ....... 261

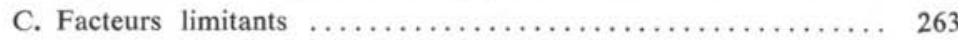

D. Vicissitudes de l'évolution des populations de bulins ....... 265

V. Biologie de B. truncatus et bilharziose :

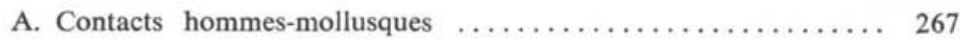

B. Pouvoir infectant des bulins .......................... 269

C. Corrélation entre distribution des bulins et distribution de la

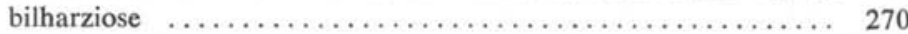

VI. Conclusions :

A. Résumé des données récoltées sur la bibologie des bulins ..... 272

B. Déductions quant à la prévention de la bilharziose ........ 274 
En 1958, le Gouvernement iranien et l'Organisation mondiale de la Santé ont conçu un projet commun de lutte contre la bilharziose dans la province iranienne du Khouzistan. Après une étude préalable de trois mois, à la fin de l'année 1958, un programme fut mis sur pied, dont l'exécution commença en 1959 et se poursuit actuellement. La masse des documents recueillis au cours de cette exécution fera sans doute un jour l'objet d'une publication d'ensemble. Nous avons seulement réuni ici les observations faites sur la biologie et l'écologie de Bulinus truncatus au cours de la mission d'études préalables et des deux premières années de réalisation du programme. Nous tenons à rendre ici hommage à l'Institut de Parasitologie de l'Université de Téhéran, pionnier des études sur la bilharziose en Iran, sans le support duquel notre travail n'aurait pu être fait. Nous remercions spécialement le $\mathrm{D}^{r} \mathrm{Ch}$. Mofidi, Directeur par intérim, et le $\mathrm{D}^{r}$ Bijan, Directeur-adjoint de l'Institut de Parasitologie de Téhéran, auprès de qui nous avons trouvé compréhension, aide matérielle, conseils précieux et témoignages d'amitié.

\section{Introduction}

\section{A. Données générales.}

La bilharziose urinaire, à Schistosoma haematobium, est seule connue en Iran, où elle paraît localisée à la plaine du Khouzistan. Cette dernière est la prolongation orientale de la Mésopotamie. Elle s'étend de la frontière artificielle Iraq-Iran jusqu'à la chaîne des monts Zagros et débouche, au Sud, sur le Golfe Persique.

C'est une vaste plaine alluviale, au sous-sol assez fortement salé. Son climat est semi-aride. La température présente de fortes variations au cours du nyctémère, comme de fortes variations au cours des saisons. Les maxima d'été atteignent et dépassent $50^{\circ} \mathrm{C}$, les minima d'hiver sont aux alentours de $0^{\circ} \mathrm{C}$. L'humidité relative est basse, surtout en été, lorsque les vents soufflent principalement du Nord et du Nord-Est. Elle s'élève lorsque le vent vient du Sud, éventualité plus fréquente en hiver. La pluviométrie est très variable d'une année à l'autre et d'un lieu à l'autre, beaucoup de précipitations revêtant l'aspect d'orages locaux. La saison des pluies est pratiquement limitée à la période novembre-avril. La hauteur moyenne des précipitations est faible, de l'ordre de 350 à $400 \mathrm{~mm}$. La nébulosité est faible; le rayonnement solaire terrible.

Ces conditions climatiques font d'une grande partie du Khouzistan une steppe sub-désertique. Mais la plaine est traversée par des cours d'eau importants, qui drainent les pentes Sud-Ouest de la haute chaîne des monts Zagros. Grâce à ces cours d'eau et aux réseaux d'irrigation qui en dérivent, de vastes 
zones de la plaine sont largement arrosées. Cette juxtaposition d'un climat semi-aride et d'une relative abondance des eaux de surface fait du Khouzistan un pays de saisissants contrastes. On y trouve des dunes de sable non loin de grands marécages. Une faune sauvage xérophile (gazelles, gangas, varans, uromastix) est survolée par une exceptionnellement riche avifaune aquatique (cf. p. 7). Les rizières alternent avec les champs de blé. Dans les troupeaux rentrant le soir aux villages, les buffles voisinent avec les moutons et les chèvres. La boue autant que la poussière complique les parcours sur les difficiles routes et pistes du Khouzistan.

La population de la plaine du Khouzistan est surtout rurale, adonnée à l'agriculture et à l'élevage. Elle comprend au moins deux éléments ethniques différents (carte 5): des Arabes dans le Sud, des populations proprement iraniennes dans le Nord. Celles-ci appartiennent à deux grandes tribus distinctes : Lur à l'Ouest, Bakhtiari à l'Est. La densité humaine est forte dans les zones irriguées, faible ou très faible ailleurs. Les cultures principales sont le blé et l'orge, produits avec peu ou pas d'irrigation, le riz, le sésame et les fèves. Les terres sont sous un régime de grande propriété. Leur rendement actuel est faible, tant à cause de pratiques agricoles archaïques qu'à cause de l'insuffisance de l'eau en été. En dehors des zones pétrolifères, l'équipement économique et social du pays est médiocre.

Les enquêtes de l'Institut de Parasitologie de Téhéran et celles du Dr Gremliza, médecin épidémiologistes travaillant pour le * Khouzistan Development Service », ont fait connaître la répartition générale actuelle de la bilharziose urinaire au Khouzistan. Une zone infectée centrale, d'environ $2.000 \mathrm{~km}^{2}$, s'étend entre les villes de Dizful et d'Ahwaz, autour des vallées des rivières Diz et Shahur (carte 1). Une zone infectée de moindre surface occupe les basses terres où se perd le fleuve Karkheh. Des foyers ectopiques entourent ces deux zones, au Nord, à l'Est et au Sud. La prévalence de l'infection est très variable d'un village à l'autre et, probablement, d'une année à l'autre. Le total des personnes actuellement infectées doit être de l'ordre de 100.000 (carte 1).

Le Khouzistan est actuellement le siège d'un vaste programme de développement comprenant, entre autres, l'édification d'un grand barrage sur l'une de ses rivières, le Diz. Ce barrage est destiné à fournir de l'énergie électrique d'une part, à étendre les irrigations d'été d'autre part. Cette extension des irrigations dans une zone infectée de bilharziose est évidemment susceptible de donner un coup de fouet à cette parasitose, jusqu'ici assez bien tolérée par les populations atteintes.

C'est pour choisir les méthodes les mieux adaptées à la prévention de la bilharziose dans les conditions propres au Khouzistan qu'a été entreprise la présente étude sur la biologie de $B$. truncatus, hôte intermédiaire de $S$. haematobium en Iran. 
CARTE I. - Carte des foyers de bilharziose urinaire du Khouzistan.

Le secteur étudié couvre la moitié nord de l'aire du foyer le plus important

CARTE II. - Le secteur étudié. Il englobe, en plus de la moitié nord du foyer central de bilharziose, la zone pilote d'irrigation pérenne et la Sugar Cane area, zone de plantations expérimentales de cannes à sucre

CARTE III. - Répartition de B. truncatus et origine des eaux d'irrigation. La répartition des bulins ne montre aucune différence statistiquement significative entre les zones irriguées par des eaux en provenance de cours d'eaux différents

CARTE IV. - Répartition de B. truncatus et bassins drainants. La répartition des bulins ne montre aucune différence statistiquement significative entre les différents bassins drainants

CARTE V. - Répartition de la bilharziose et ethnologie humaine. Les foyers d'infection bilharzienne sont localisés sur les territoires de tribus arabophones et des tribus Bakhtiari, chez qui de nombreux immigrants arabes se sont implantés

Ann. Parasito, hum. et comp.

tome $37, n^{\circ} 3$, avril-juin 1962

Masson et $C^{\text {le }}$, Editeurs 


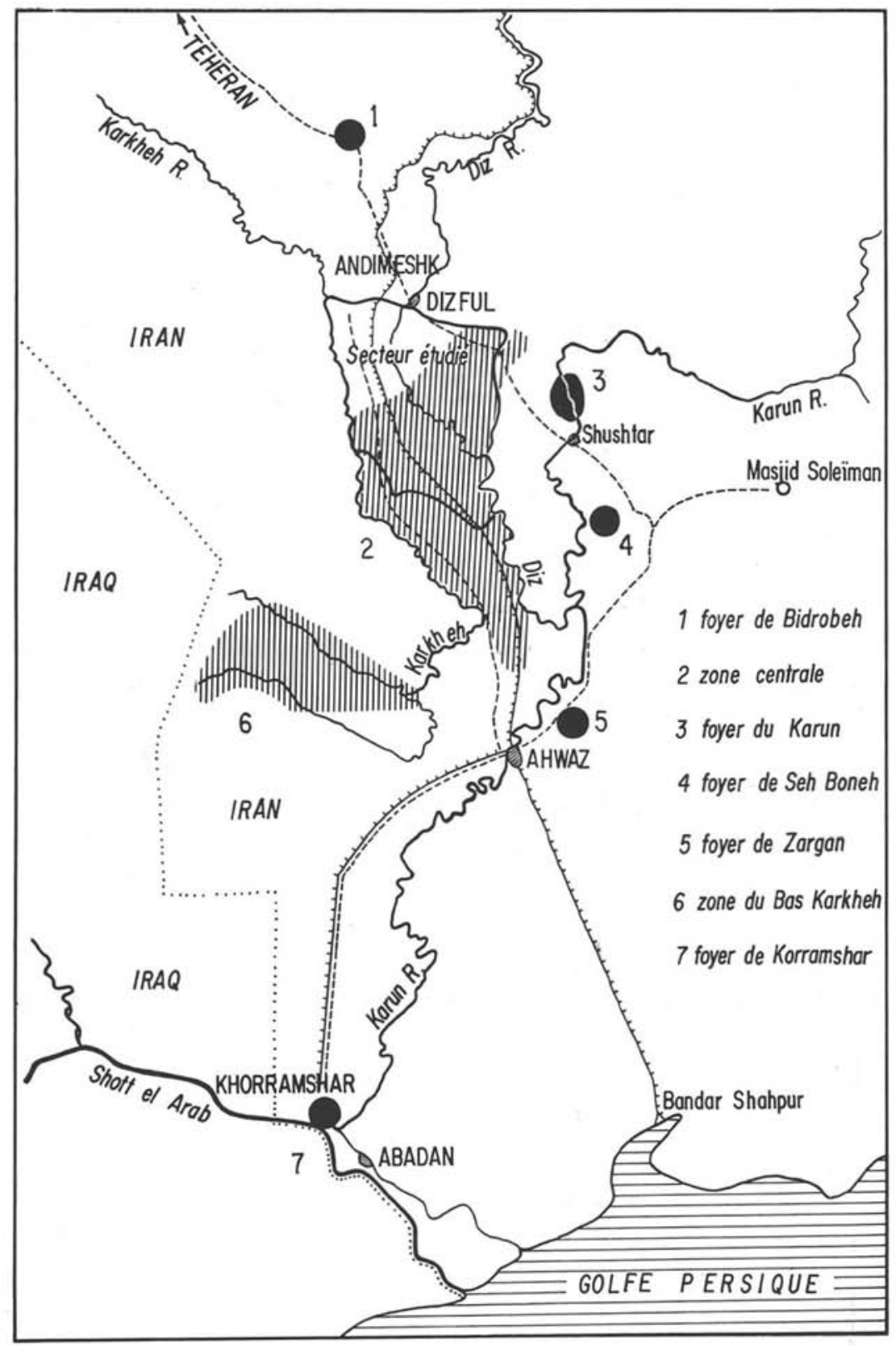

Carte 1

Ann. Parasito. hum. et comp. tome 37, $n^{\circ} 3$, avril-juin 1962 


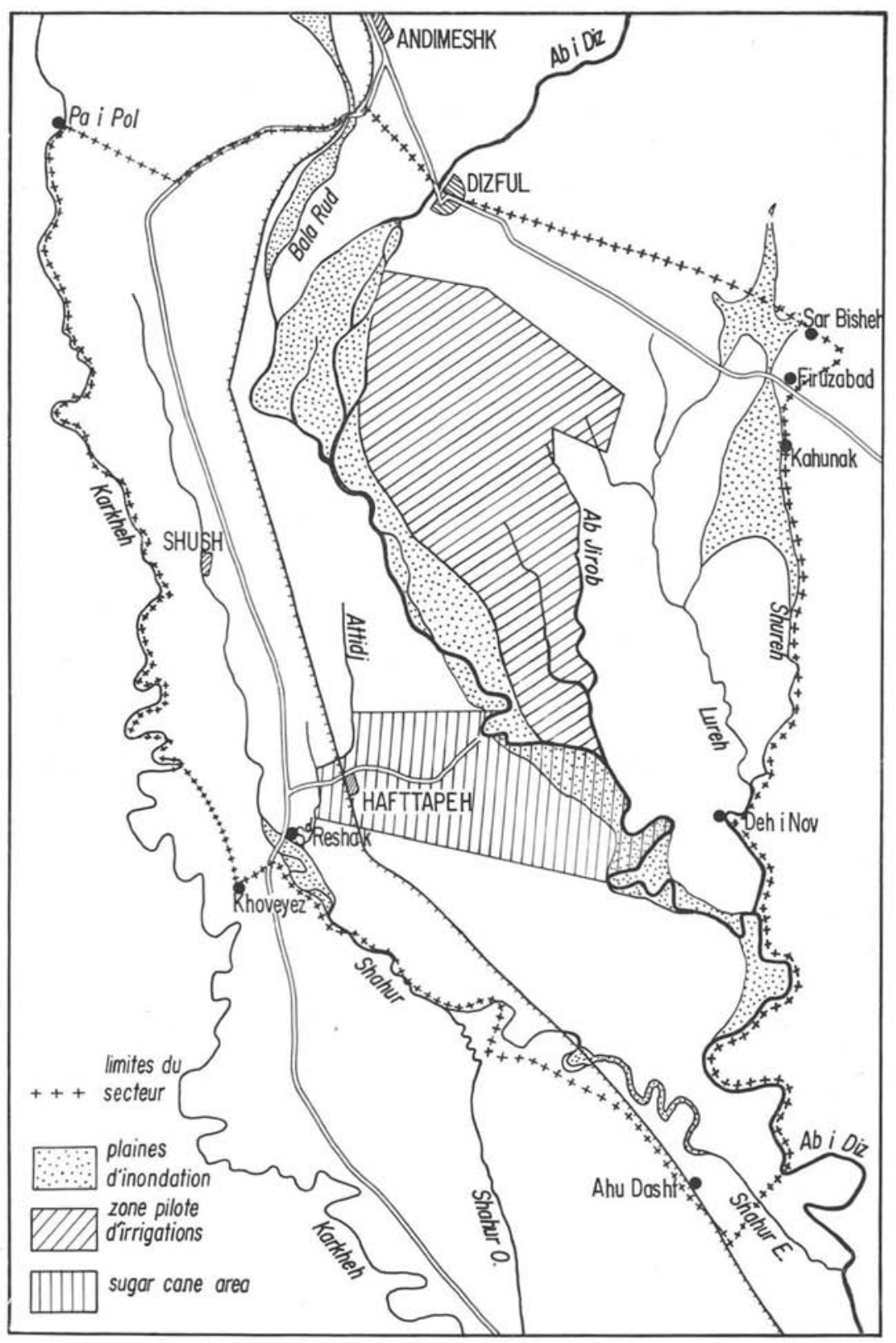

CARTE 2

Ann. Parasito. hum. et comp. tome $37, n^{\circ} 3$, avril-juin 1962 


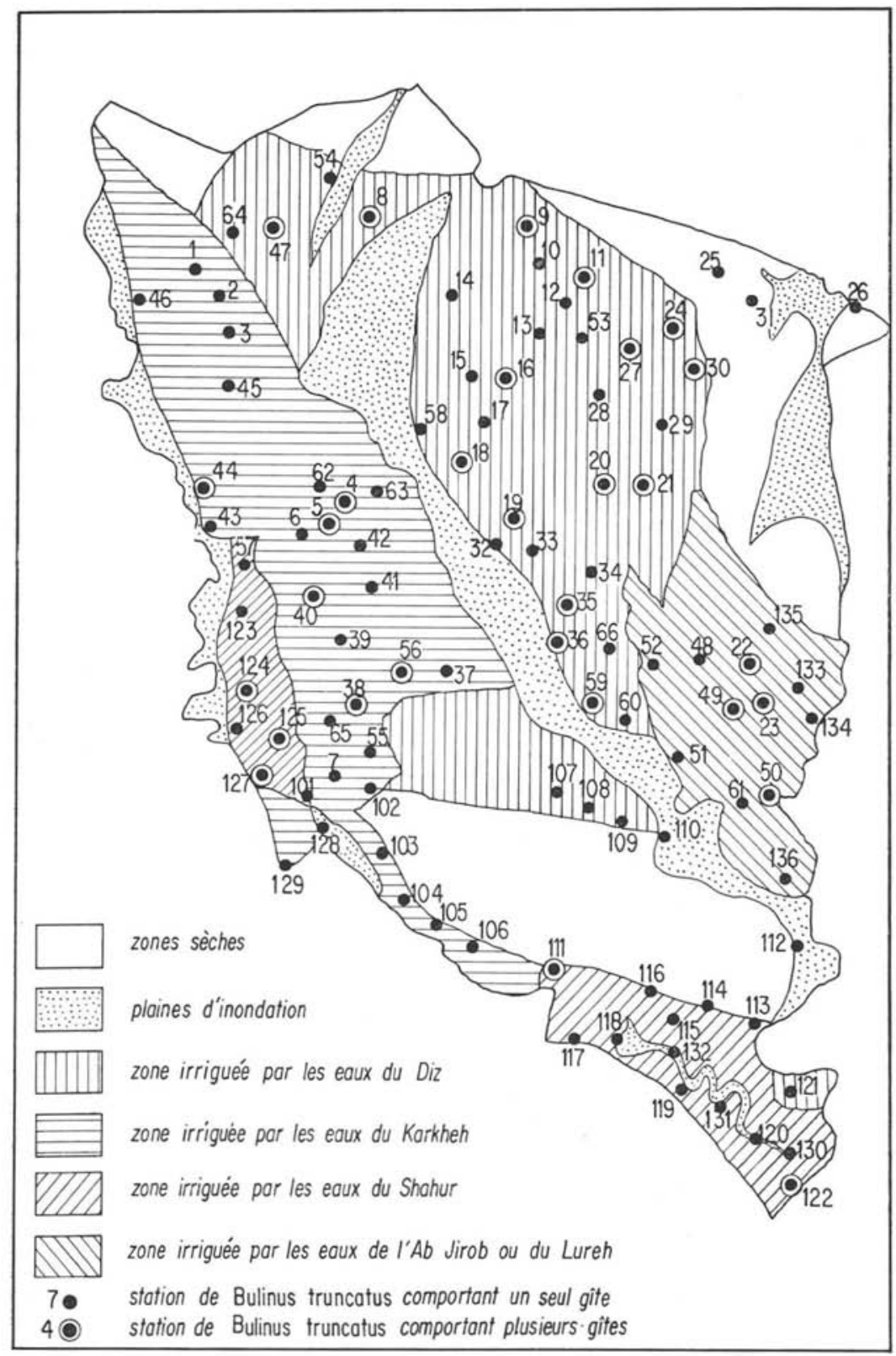

CARTE 3

Ann. Parasito. hum. et comp. tome 37, $n^{\circ} 3$, avril-juin 1962 


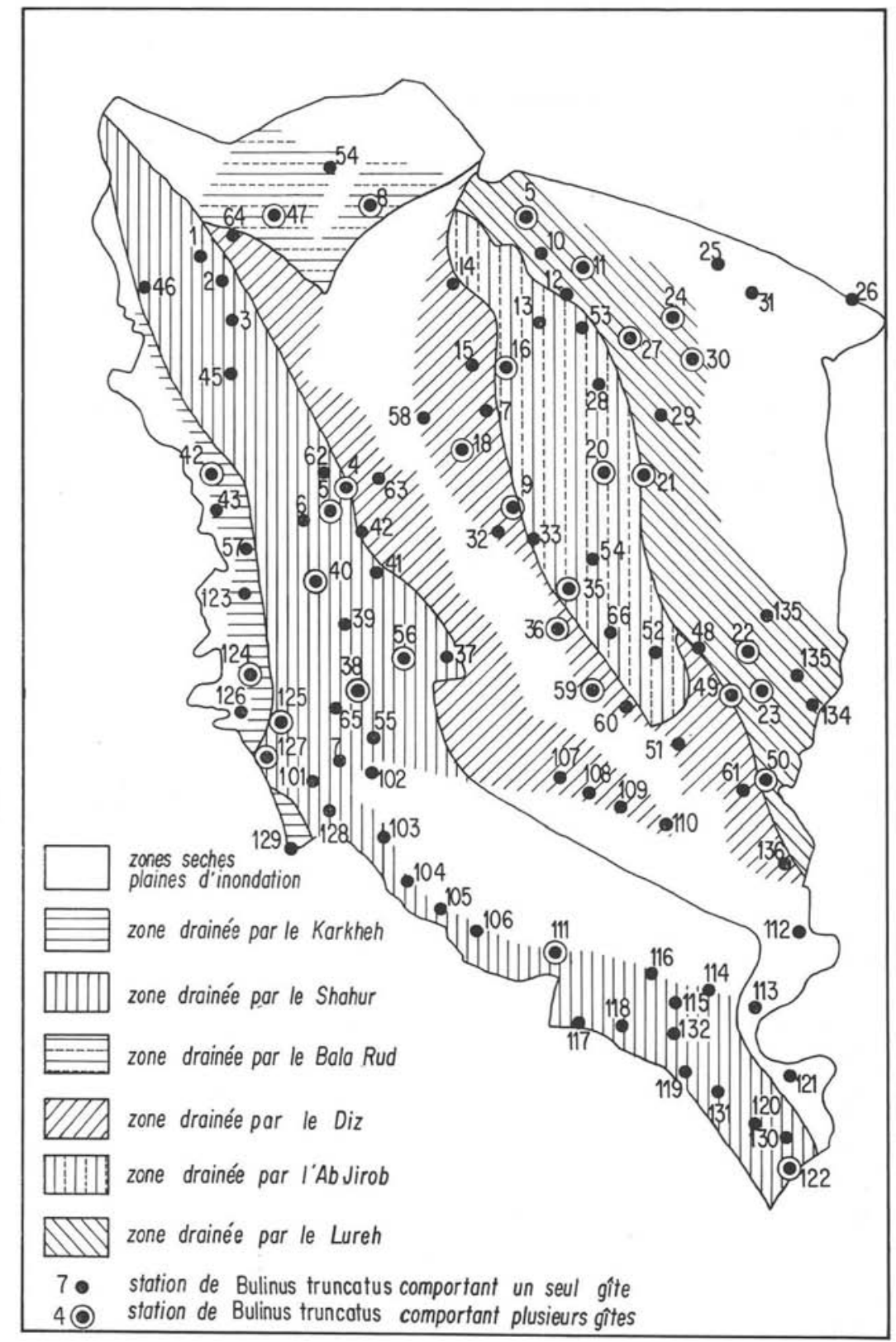

Carte 4

Ann. Parasito. hum et comp.

tome 37, $n^{\circ} 3$, avril-juin 1962

Masson et $\mathrm{C}^{\text {ie }}$, Editeurs 


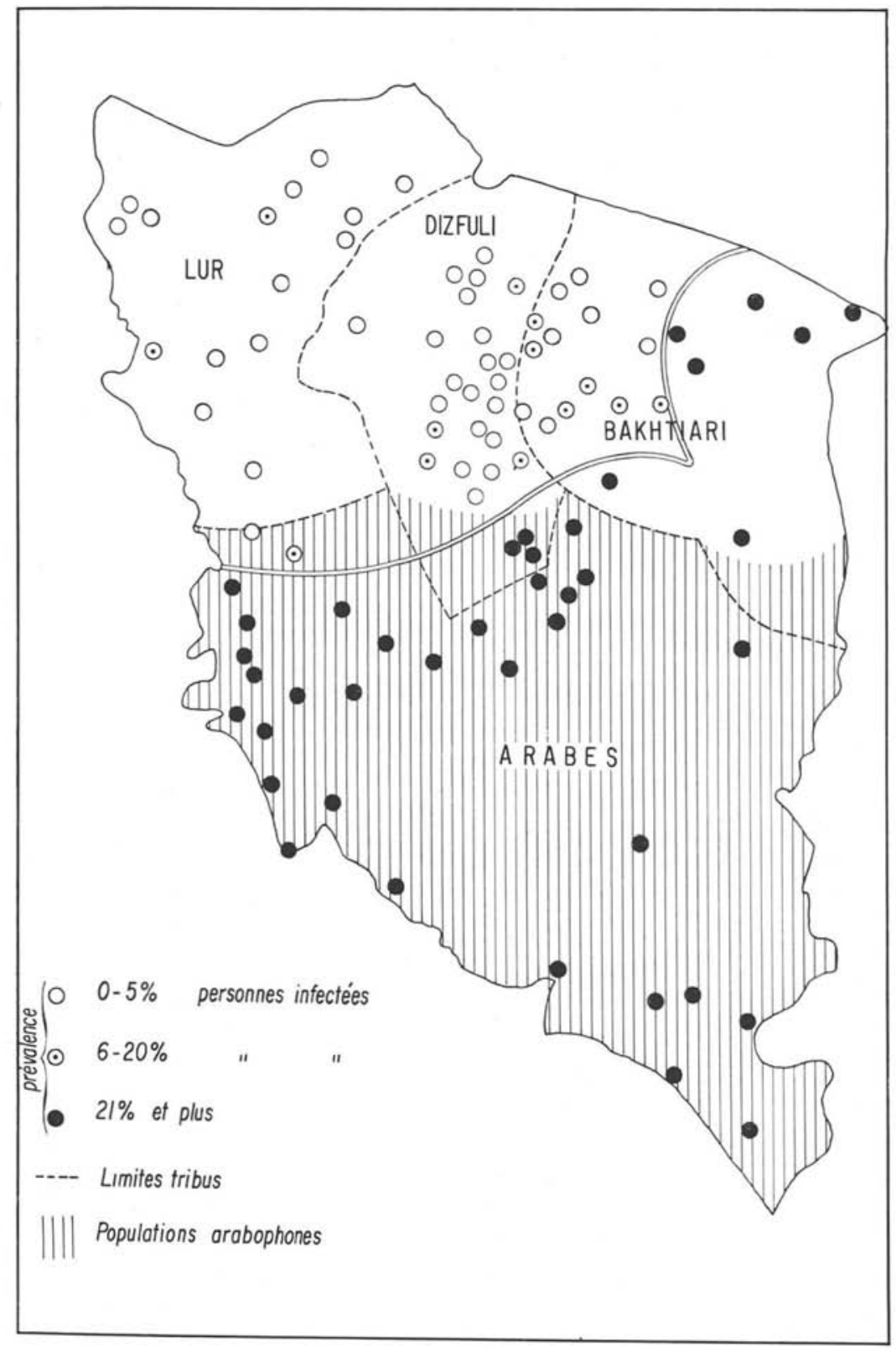

CARTE 5

Ann. Parasito. hum. et comp.

tome $37, n^{\circ} 3$, avril-juin 1962

Masson et $\mathrm{C}^{\text {ie, }}$, Editeurs 


\section{B. Le secteur d'étude.}

Les conclusions présentées dans ce rapport sont basées sur les observations faites dans un secteur d'étude limité.

\section{Choix du secteur d'étude.}

Le secteur où ont été faites les observations sur l'écologie des mollusques, et où devront ensuite être entreprises les expériences de lutte contre ceux-ci, a été choisi en fonction de divers imperatifs:

- Ce secteur devait être situé à proximité de la ville de Dizful, qu'une étude préliminaire avait montré être la meilleure base d'opérations possible. Il devait être d'accès facile et rapide sur toute sa surface à partir de cette base.

- Il devait être représentatif de l'ensemble de la zone touchée ou menacée par la bilharziose et présenter une variété de conditions (hydrographiques, agricoles, etc.) reproduisant les diverses situations existant dans cette zone. Ainsi, seulement, les observations faites dans le secteur seraient utilement extrapolables hors dé célui-ci.

- Il devait inclure la zone-pilote où doit être d'abord mise en œuvre l'irrigation pérenne. Il devait également inclure la * Sugar cane area », zone de plantations expérimentales de canne à sucre, culture qu'on espère voir se développer dans la province.

- Il devait inclure une partie au moins de la vallée du Shahur, zone que les enquêtes préalables menées par l'Institut de Parasitologie de Téhéran indiquaient comme particulièrement affectée par la bilharziose.

Ces conditions, parfois contradictoires, sont à peu près réunies dans le secteur d'étude finalement choisi, qui couvre une surface de $1.647 \mathrm{~km}^{2}$ au Sud de la ville de Dizful.

\section{Description générale du secteur d'étude.}

Ce secteur (carte 2) s'inscrit dans un quadrilatère irrégulier, de forme vaguement losangique. Le grand axe, N.O.-S.E., mesure $73 \mathrm{~km}$. depuis le bac sur le Karkheh de $\mathrm{Pa}$ i Pol, jusqu'à $3 \mathrm{~km}$. au Sud de la station de chemin de fer d'Ahu Dasht. Le petit axe, N.E.-S.O., mesure $45 \mathrm{~km}$. depuis $3 \mathrm{~km}$. à l'Est du village de Sar Bisheh jusqu'à Khoveyez.

Dans la mesure du possible, on a choisi à ce secteur des limites repérables. Au Nord (d'Ouest en Est) : route du bac du Karkheh à Dizful ; piste de Dizful à Sar Bisheh. A l'Est (du Nord au Sud) : route de Sar Bisheh à Firouzabad; rive gauche de la grande plaine d'épandage de Kahunak; cours de la rivière Shureh; cours du Diz. A l'Ouest : cours de la rivière Karkheh, puis route longeant le canal de la S.A. $C^{\circ}$ jusqu'à Khoveyez; au Sud, enfin (du Nord-Ouest 
au Sud-Est), route de Khoveyez à Sheikh Reshak; cours du Shahur Nord jusqu'à Mianab ; route de Mianab à Ahu Dasht ; voie ferrée sur $3 \mathrm{~km}$. au Sud de cette station.

Le secteur d'étude est traversé par la grande route de Téhéran à Ahwaz dans sa partie Ouest, par la route de Dizful à Shushtar dans sa partie NordEst. En dehors de ces deux axes, de nombreuses pistes permettent l'accès par automobile d'à peu près toute la surface du secteur. Ces pistes ont malheureusement le défaut de ne pas constituer un réseau. Faute d'anastomose, il est souvent nécessaire de faire de longs détours pour passer d'un village à un autre village très proche. Ces pistes sont toujours éprouvantes pour le matériel roulant; elles sont souvent imprévisiblement coupées par un canal nouvellement creusé ou par de trop généreuses irrigations. La voie ferrée Téhéran-Khorramshar traverse du Nord au Sud la partie Ouest du secteur.

Sur l'ensemble du secteur, les reliefs sont faibles. La pente générale est NordSud, assez forte. On rencontre trois faciès principaux. Les plaines d'inondation des rivières (cf. chapitre suivant) couvrent $195 \mathrm{~km}^{2}$, soit $1 / 8^{e}$ de la surface totale. Les zones sèches, portant des cultures de céréales dans le Nord du secteur, surtout utilisées dans le Sud comme terrains de parcours des troupeaux, couvrent $351 \mathrm{~km}^{2}$, soit $1 / 5^{\circ}$ environ de la surface totale du secteur. Les terres irriguées couvrent presque les $2 / 3$ du secteur : $1.011 \mathrm{~km}^{2}$; elles constituent un ensemble agricole assez homogène, la Sugar cane area mise à part.

La zone-pilote où sera d'abord appliquée l'irrigation pérenne (bande allongée N.S. à l'Est du Diz, d'une superficie de $250 \mathrm{~km}^{2}$ ) est incluse entièrement dans le secteur. Elle en occupe à peu près la partie axiale et représente $1 / 6^{\circ}$ environ de sa surface totale, soit 1/4 de la surface irriguée. La Sugar cane area (bande allongée E.O. à l'Ouest du Diz) est entièrement incluse dans la moitié Sud du secteur. Elle occupe $1 / 20^{\circ}$ de la surface totale de celui-ci, soit $1 / 12^{\circ}$ de la superficie irriguée.

La densité humaine, dans le secteur d'étude, est élevée, particulièrement dans la partie Nord-Ouest (entre 60 et 70 habitants au $\mathrm{km}^{2}$ ). La population est concentrée en villages compacts de 250 à 1.000 habitants. On compte 195 de ces villages dans l'ensemble du secteur, dont 50 dans la zone-pilote d'irrigation. La population totale peut être estimée entre 75.000 et 80.000 habitants.

Le secteur est assez représentatif des conditions hydrographique et agricoles existant au Khouzistan. Cependant, les pentes y sont plutôt plus fortes, la perméabilité du sol plus grande, les terrains et les eaux moins salés que cela ne s'observe dans le reste de la zone infectée ou menacée par la bilharziose ; la densité humaine y est aussi plus élevée. Tout ceci est plus particulièrement vrai dans la moitié Nord du secteur. 


\section{Aspects écologiques généreux}

\section{A. Hydrographie.}

\section{Rivières et grands drains naturels.}

A l'exclusion de quelques petites sources, situées dans l'angle N.E. du secteur, les eaux proviennent de deux grandes rivières descendant des pentes S.O. de l'extrémité Nord des monts Zagros. Le Karkheh dessine la bordure Ouest du secteur; son bassin d'alimentation couvre $42.000 \mathrm{~km}^{2}$ et son débit moyen à $\mathrm{Pa}$ i Pol est de $143 \mathrm{~m}^{3} /$ seconde. Le Diz coupe le secteur, du Nord au Sud, en deux parts presque égales. A $10 \mathrm{~km}$. au Sud de Dizful, il reçoit sur sa rive droite un affluent, le Bala Rud, torrent dont le cours aérien tarit complètement en été. Le bassin d'alimentation du Diz couvre $18.000 \mathrm{~km}^{2}$ et son débit moyen est de $287 \mathrm{~m}^{3} /$ seconde à Dizful.

De ces rivières partent un grand nombre de canaux d'irrigation (voir paragraphe suivant). Les eaux d'irrigation en excès, comme les eaux qui ruissellent après les pluies, se frayent tant bien que mal une voie vers les drains naturels. Ces drains, qui coulent généralement dans une direction N.S., sont : le Shahur et son affluent l'Attidj, entre Karkheh et Diz; l'Ab e Jirob, le Lureh et le Shureh, à l'Est du Diz. Le Shureh borde le côté Est du secteur étudié. Vers l'aval, ces drains prennent assez rapidement l'aspect de rivières et, avant de se jeter dans le Diz, donnent naissance à de nouveaux réseaux d'irrigation.

Tous ces cours d'eau, grandes rivières comme drains, ont un régime semitorrentiel très irrégulier, avec des variations du simple au décuple. Leur cours est sinueux et indécis, épanoui souvent en plaines d'inondation dont la largeur atteint plusieurs kilomètres. Le lit même des cours d'eau, généralement pierreux, est parcouru par un courant rapide. Les plaines d'inondation offrent des aspects très divers. Celles de la partie Nord et Est du secteur (Bala Rud, Diz supérieur, Lureh, Shureh) sont de vastes étendues sèches couvertes d'un épais matelas de galets. Celles du Karkheh et du Diz dans sa partie Sud sont en majorité couvertes d'une végétation arbustive (Tamarix) sur sol relativement humide, avec de grandes mares d'eau stagnante dans les bras morts des rivières. Au Sud, la plaine d'inondation du Shahur est beaucoup plus étroite, atteignant rarement $1 \mathrm{~km}$. de large. Elle est franchement marécageuse, avec une végétation herbacée (cf. clichés 1,2 et 3 ).

\section{Irrigations.}

Les deux tiers de la surface du secteur sont couverts par les mailles d'un vaste ensemble de réseaux d'irrigation. Il existe une cinquantaine de canaux primaires sur le secteur : 32 provenant du Diz, 6 du Karkheh, 4 du Shahur, 1 de l'Ab e Jirob et 6 du Lureh. Chaque canal appartient à un propriétaire 
ou à un groupe de propriétaires. A l'exclusion de deux stations de pompage dans le Diz, dont l'une de grand débit destinée à assurer l'irrigation de la Sugar cane area, les prises d'eau se font par simple diversion. Il n'existe pas d'ouvrages permettant de régler les débits en tête de canal. Six canaux primaires en provenance du Diz sont souterrains dans leur partie amont (Ganat); les autres sont aériens. Le débit des canaux primaires et l'étendue des secteurs dont ils assurent l'irrigation sont extrêmement différents d'un canal à l'autre. La plupart des canaux primaires desservent des surfaces de 1.000 à 3.000 hectares avec un débit maximum en tête de l'ordre de $2 \mathrm{~m}^{3} / \mathrm{seconde}$. Mais le canal Harmushi, en provenance du Karkheh, peut écouler $11 \mathrm{~m}^{3} / \mathrm{seconde}$ en tête et irrigue 20.000 hectares de terres, certaines situées à $35 \mathrm{~km}$. de l'origine du canal.

Tous les canaux, primaires, secondaires, tertiaires ou quaternaires, sont en terre. Il n'existe pas de bassins d'accumulation et de compensation et, pratiquement, pas d'ouvrages partiteurs cimentés. Le cours des canaux est souvent extrêmement sinueux. Les courants sont rapides dans la moitié Nord-Est du secteur, de 100 à $50 \mathrm{~cm}$./ seconde (moyenne : $63 \mathrm{~cm} . /$ seconde) dans les canaux primaires et secondaires, de 60 à $25 \mathrm{~cm}$./seconde (moyenne : $36 \mathrm{~cm} . /$ seconde) dans les canaux tertiaires et quaternaires. Dans la moitié Sud-Est du secteur, les pentes sont moindres et le courant des canaux moins rapide.

L'ensemble des ramifications émanant d'un seul canal primaire ne constitue pas un système clos indépendant. Il existe entre les réseaux un grand nombre d'anastomoses voulues, qui permettent aux propriétaires de faire des échanges d'eau selon leurs besoins momentanés. Les eaux en excès provenant d'un système tombent généralement dans les canaux d'irrigation d'un autre système avant d'avoir trouvé leur voie vers les drains naturels. Les réseaux sont fréquemment enchevêtrés et les canaux appartenant à des systèmes différents se croisent par aqueducs sommairement construits et rarement étanches. Les eaux de pluie, lorsqu'elles ruissellent, sont drainées par les canaux d'irrigation. Les talwegs, généralement secs, sont traversés par les canaux sans ouvrages de protection suffisants. Lors de grandes pluies, les canaux sont souvent emportés à ces points de croisement. Tout ceci amène, temporairement au moins, des anastomoses supplémentaires entre les réseaux.

Le débit des canaux est au maximum en automne et au printemps. En fin d'hiver, les canaux sont mis à sec pour curage et réparation des dégâts causés par les pluies. Les importantes conséquences de cette interruption des irrigations, pendant un à deux mois, sur la biologie des bulins, seront analysées plus loin.

Les surfaces cultivées en été sont réduites, la demande d'eau à l'unité de surface devenant plus forte alors que le débit des canaux diminue. Cette situation est appelée à se modifier. La retenue des eaux du Diz par le grand barrage actuellement en construction permettra d'augmenter les débits d'été et d'arriver 
à une irrigation pérenne. La zone-pilote d'irrigation sera la première à bénéficier de cette modification.

\section{B. Limnologie.}

\section{Données physico-chimiques.}

La température des eaux de surface, qu'elles soient courantes ou stagnantes, est beaucoup plus stable que la température extérieure. En hiver, les rapports entre la température de l'eau et celle de l'air dépendent de la nébulosité. $\mathrm{Si}$ celle-ci est forte, température de l'air et température de l'eau sont égales, constantes toutes deux au cours du nyctémère, et assez élevées (au-dessus de $10^{\circ} \mathrm{C}$ ). Lorsque la nébulosité est faible - éventualité la plus fréquente - l'insolation compense, durant la journée, les effets de l'évaporation, et la température de l'eau dépasse un peu celle de l'air; pendant la nuit, l'humidité relative s'élève, entravant l'évaporation, et les minima de température observés dans l'eau restent de 4 à $5^{\circ} \mathrm{C}$ au-dessus des minima observés dans l'air. Ces derniers sont d'ailleurs exceptionnellement inférieurs à $0^{\circ}$; le minimum absolu de l'hiver 1960-1961 a été de $3^{\circ}$ le 24 janvier 1961.

En été, la température de l'eau reste inférieure à celle de l'air pendant tout le nyctémère. Pendant la journée, en particulier, l'extrême sécheresse atmosphérique entraîne une très forte évaporation qui compense largement les effets de l'insolation; les maxima de température observés dans l'eau restent inférieurs à $40^{\circ}$ — et dépassent rarement $37^{\circ}$ - alors que la température de l'air à l'ombre atteint et dépasse $50^{\circ}$.

Les températures maxima et minima relevées mensuellement dans différents gîtes à bulins font l'objet du tableau $\mathrm{I}$.

Les eaux de surface du Khouzistan ont un $p \mathrm{H}$ généralement supérieur à 7 : de 7 à 8 pour les eaux courantes des rivières et des canaux, de 7 à 8,5 pour les eaux stagnantes. Dans ces dernières, le $p \mathrm{H}$ varie dans le temps. Il est plutôt plus élevé en hiver, avec toutefois des chutes brusques à 7-7,5 après les grandes pluies.

La salinité est assez forte. La teneur globale en sels varie, dans les cours d'eau, de $340 \mathrm{mg}$. par litre (Diz à Dizful) à $950 \mathrm{mg}$. par litre (Lureh). Elle dépasse $2.000 \mathrm{mg}$. par litre dans beaucoup d'eaux stagnantes (marécages de B. Djavaz et de Deh Now, à l'Est du secteur). Le tableau II ci-après, résumant une quarantaine d'analyses, donne une idée des compositions observées et de la tolérance de $B$. truncatus à divers éléments chimiques.

Les eaux sont généralement dures. La dureté totale est comprise entre 135 et 396 dans les analyses faites. L'alcalinité, exprimée en p.p.m. de $\mathrm{CO}^{3} \mathrm{Ca}$, varie de 100 à 187 dans les mêmes analyses.

Les eaux courantes sont très chargées en particules argileuses. Les eaux stagnantes se clarifient lentement si elles ne sont pas remuées. Pratiquement, 


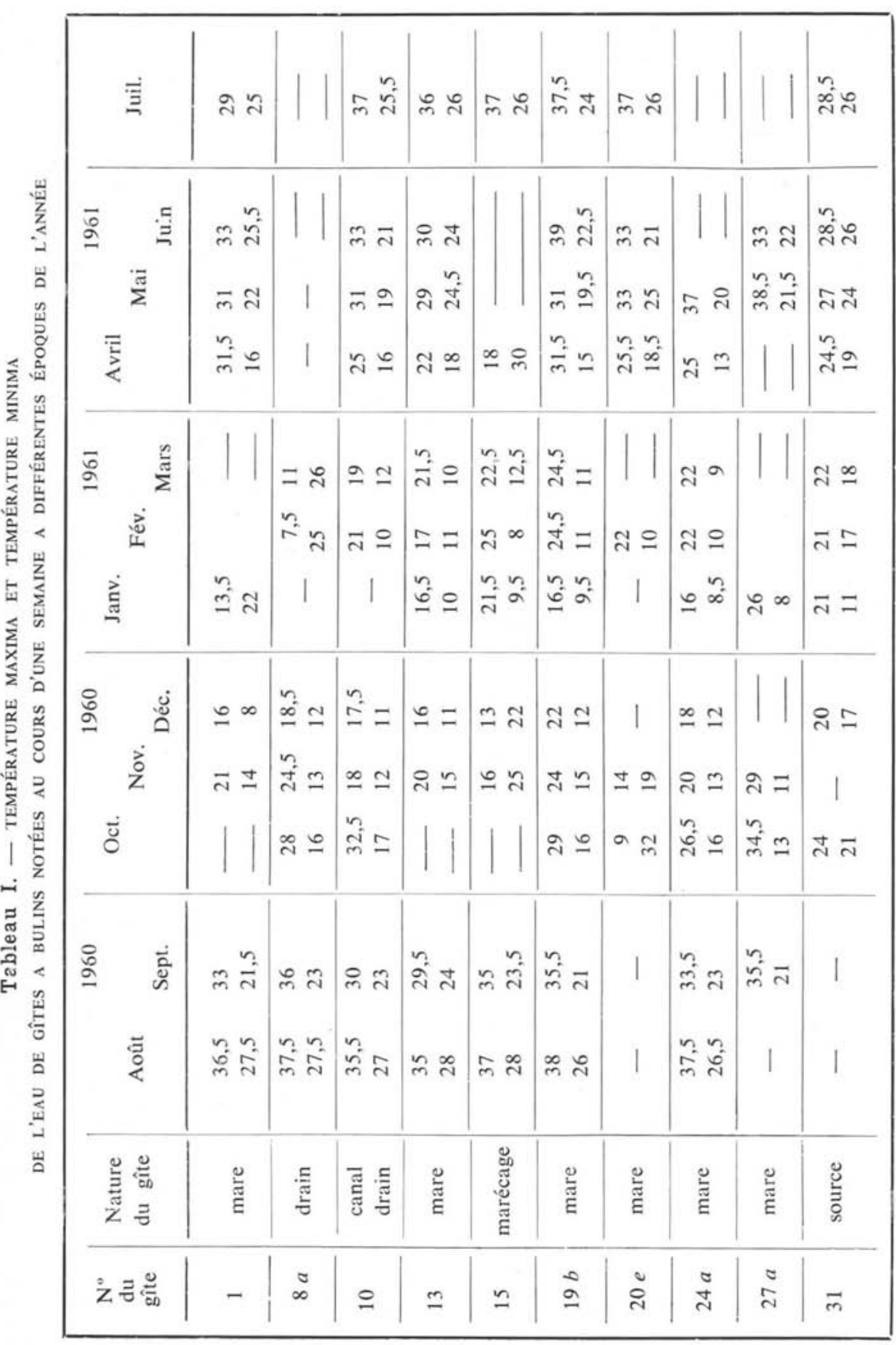

ச்

苟 $\mid$ กิ 


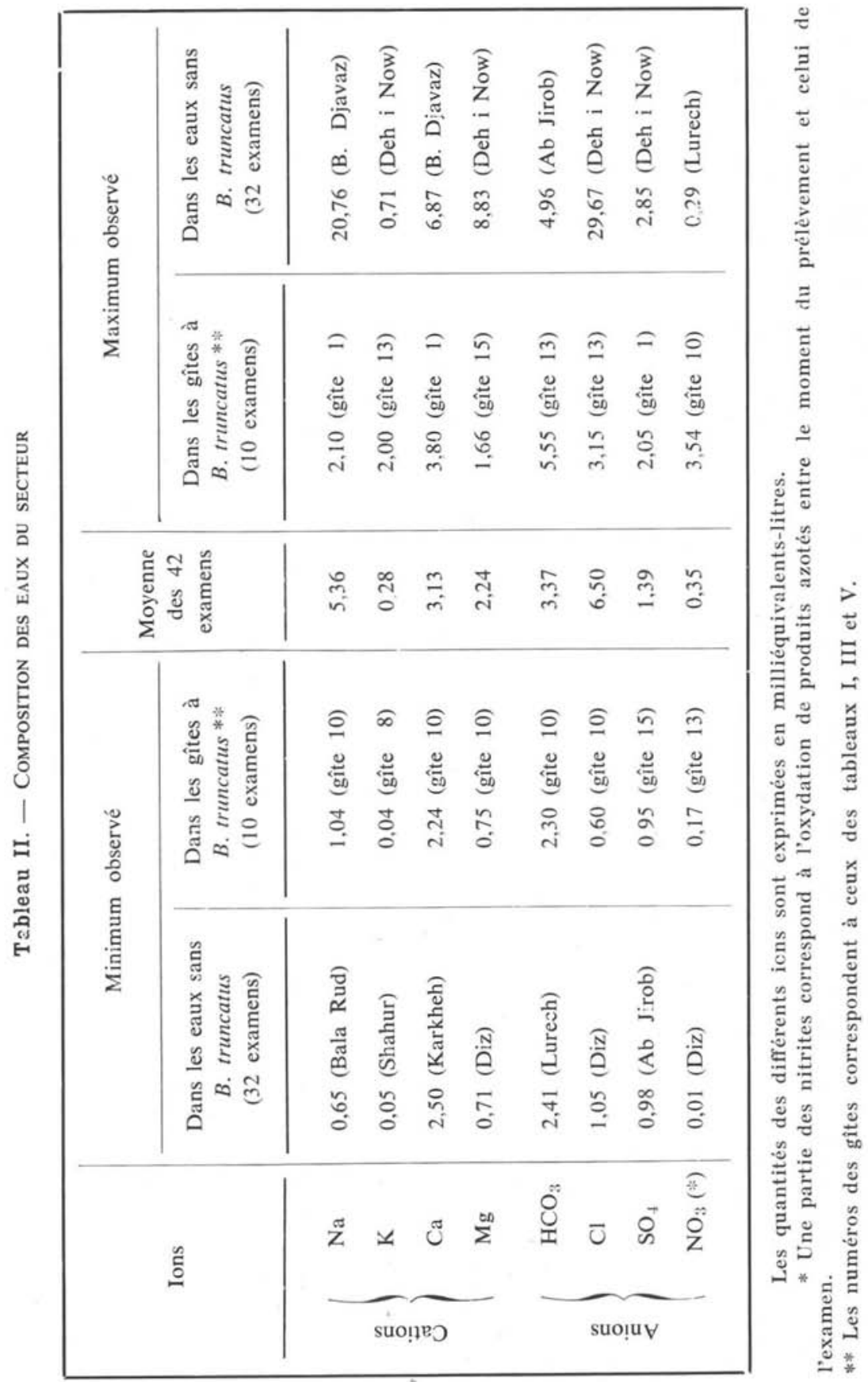


le simple mouvement des animaux suffit à maintenir en suspension une notable quantité d'argile et la turbidité est forte.

\section{Flore et faune.}

Toutes les eaux stagnantes ou faiblement courantes sont rapidement envahies par une abondante végétation aquatique. La végétation verticale émergeante est particulièrement abondante. Les groupes les mieux représentés sont les Typha, les scirpes, les joncs et divers genres de chiendents. Les roseaux sont rares et d'assez maigre venue. Les Polygonum sont souvent rencontrés sur les bords de l'eau, plus ou moins immergés. Par contraste, la végétation flottante est très pauvre. On rencontre rarement des Potamogeton, exceptionnellement des Lemna ou des Salvinia. La végétation macroscopique immergée est irrégulière, parfois absente, parfois très abondante et formant feutrage. Les groupes les plus souvent rencontrés sont les zonichielles en hiver, les renoncules au printemps, les Ceratophyllum et les characées en été et en automne (cliché 7). On rencontre aussi diverses algues filamenteuses et, plus rarement, des Hydrodyction.

Une étude du phytoplancton n'a malheureusement pas pu être faite. Ceci est d'autant plus regrettable qu'on a pu observer le développement normal des bulins dans les eaux absolument dépourvues de végétation macroscopique (Gîte d'Ab Koweit, $\mathrm{n}^{\circ}$ 31, cliché 5). Par ailleurs, à Téhéran, a pu être observée la destruction de toutes les pontes d'un élevage de bulins par une diatomée du genre Pinularia. La diatomée n'attaquait pas les embryons eux-mêmes, mais sa prolifération massive dans la gelée périovulaire était fatale à ceux-ci. Ces faits montrent l'importance du phytoplancton dans l'écologie des bulins.

La faune aquatique est abondante, mais constituée surtout de groupes dont l'habitat dulcaquicole est limité à une phase de l'existence, comme les têtards d'amphibiens et les larves de diptères et d'odonates. Les hémiptères (hydromètres, nautonectes, naucores, belostomes) sont abondamment représentés ; les coléoptères beaucoup moins (ditiques). Les petits crustacés sont très abondants, particulièrement Ostracoda, Conchostraca et Cladocera (daphnies). Les crevettes se rencontrent seulement dans les grands marécages permanents. Les hydracariens sont nombreux également. Les sangsues sont très rares.

Les vertébrés proprement aquicoles sont représentés par d'assez rares poissons. Ceux rencontrés dans les petites collections d'eau stagnante semblent incapables d'y atteindre leur plein développement, si l'on en juge par la taille des exemplaires observés.

Par contre, la faune des vertébrés aquaphiles est extrêmement riche. Les oiseaux en constituent l'élément le plus marquant. Un grand nombre d'ordres sont représentés: Pélécaniformes (cormorans et pélicans), Ardéiformes (hérons, cigognes), Ansériformes (oies et canards), Lariformes (sternes), Ralliformes (râles, foulques), Coraciadiformes (martins-pêcheurs), mais surtout Charadriiformes (glaréoles, pluviers, vanneaux, combattants, bécasseaux, bécassines, 
échasses). En dehors des oiseaux, on note la présence de nombreuses tortues d'eau du genre Clemmys, de serpents du genre Tropidonotus. Les amphibiens, grenouilles en particulier, sont extraordinairement abondants.

La faune de mollusques aquatiques est assez pauvre en espèces. Les pulmonés sont représentés par quatre espèces seulement : Bulinus truncatus, Lymnaea truncatula, Gyraulus intersitus et Lymnaea gedrosiana. Les deux dernières espèces sont rencontrées avec une très grande fréquence dans toutes sortes d'eaux courantes ou stagnantes. Parmi les prosobranches, Melanoïdes tuberculata est répandue dans des eaux assez diverses. Plusieurs espèces de Melanopsis se rencontrent dans les eaux courantes. Nous avons trouvé tout à fait exceptionnellement des néritines et des bithynies. Les Siphonommatophores (Succinea) sont très rares. Des Lamellibranches pélécypodes se rencontrent surtout dans les eaux courantes et les canaux d'irrigation.

\section{Rôle de l'homme dans l'écologie des bulins.}

Nous ne parlerons ici que des activités humaines qui nous ont paru avoir un rapport plus ou moins direct avec l'écologie des bulins. L'agriculture est évidemment la plus importante de ces activités. Nous avons décrit plus haut, sommairement, le réseau d'irrigation. Nous envisagerons dans le présent chapitre les cultures irriguées elles-mêmes. Quatre d'entre elles nous ont paru présenter un intérêt spécial : canne à sucre, jardins, rizières, maraîchage. Mais l'agriculture n'est pas la seule activité humaine ayant des rapports avec la bilharziose : nous avons noté en particulier le rôle de l'élevage, ainsi que celui de l'industrie de l'argile. Le choix des eaux utilisées pour la boisson et les usages domestiques mérite enfin considération.

\section{Canne à sucre.}

Nous n'avons jamais pu trouver de bulins dans les champs de canne expérimentaux créés il y a trois ans, en dehors - et à l'Ouest - de la Sugar cane area. Quant aux plantations de cette dernière zone, elles sont très récentes. Un large nivellement, véritable bouleversement du sol, a précédé la mise en culture, et les canaux, neufs, n'ont pas encore pris leur équilibre biologique définitif. A priori, le danger d'infestation des plantations elles-mêmes par les bulins semble faible. D'une part, les plantations sont largement irriguées, mais pas submergées ; d'autre part, l'exemple de différents pays n'est pas en faveur d'une infestation des champs de canne par les mollusques. Mais les eaux en excès, et les eaux de drainage, ont déjà constitué des gîtes en aval des plantations ( $\left.\mathrm{n}^{\circ \mathrm{s}} 107,108,109\right)$. Le nombre de ces gîtes ira-t-il croissant ? La question mérite d'être suivie avec attention.

\section{Jardins.}

Chaque village ou presque comporte, à proximité immédiate des habitations, un ou plusieurs enclos qu'il est d'usage dans la région d'appeler jardins. Ils

Ann. de Parasitologie, T. XXXVII, No $3 .-1962$. 
seraient mieux nommés vergers, car leurs hauts murs enferment essentiellement des arbres: arbres fruitiers en majeure part, mais aussi autres essences (eucalyptus, jujubiers). La superficie de ces jardins est généralement plus grande que celle du village: deux, quatre fois, ou même plus; le plus souvent, une seule porte y donne accès, rarement plus de deux. Cés portés sont soigneusement fermées, et l'entrée d'un jardin nous a été souvent interdite jusqu'à ce que nous ayons l'autorisation du propriétaire (habitant généralement Dizful),

Ces jardins sont des endroits frais, où les arbres mal taillés poussent dans un désordre parfois inextricable. Il faut souvent s'y diriger à l'aveuglette en se défendant des branches et des ronces. Les vagues sentiers forment des labyrinthes où il nous est arrivé de nous égarer. Les canaux d'irrigation forment un réseau mieux ordonné que les sentiers ; leurs branches terminales se raccordent à un ou deux drains, aboutissant à l'extérieur par des orifices percés dans le bas des murs. L'eau en excès stagne parfois dans de petites mares à l'intérieur des jardins; dans de telles collections d'eau, nous n'avons qu'une fois trouvé des bulins ( $\mathrm{n}^{\circ} 20 e$ des tableaux). Plus souvent, c'est à l'extérieur des jardins que les eaux de drainage constituent ou alimentent des mares. Ces dernières sont alors pour les bulins des gîtes très favorables.

\section{Rizières.}

Chaque village ou presque possède ses rizières, et les surfaces consacrées à la riziculture sont probablement appelées à s'accroître lorsque les projets d'irrigation pérenne se réaliseront. Le riz est semé en pépinières en juin, repiqué en juillet-août, et moissonné en fin octobre. En cours de culture, il n'y a pratiquement pas d'asséchement jusqu'un peu avant la moisson. L'eau calme, tiède et peu profonde des rizières devrait constituer, $a$ priori, un gîte favorable pour les bulins. Mais, pratiquement, nous avons trouvé des bulins deux fois seulement sur plus d'une centaine de rizières examinées. Encore était-ce dans les parties marginales des rizières, où l'eau était plus profonde et où le riz était mélangé à des scirpes, avec une végétation sous-jacente de characées ou de Ceratophyllum.

La culture du riz semble pourtant avoir indirectement une importance considérable dans le maintien des populations de bulins. Les eaux de drainage des rizières, plus encore que celles des jardins, alimentent en eau durant l'été des gîtes qui, sans elles, seraient condamnés à un asséchement meurtrier pour les mollusques.

En relation directe avec la riziculture, semble se développer une nouvelle pratique particulièrement favorable au développement des bulins. Les villageois utilisent, pour attacher les bottes de riz lors du repiquage, des feuilles de Typha (fârsi : louban ; arabe : bardi). Ces plantes croissent spontanément dans les marécages et sur les bords des cours d'eau. Nous les avons trouvées 
aussi dans plusieurs villages, peuplant des mares permanentes. Nous avons appris que les villageois avaient eux-mêmes transplanté ces Typha et en considéraient la culture comme lucrative. Les habitants de Q. Seyed (gîte $\mathrm{n}^{\circ} 13$, tableau III) ont prétendu réaliser annuellement une économie de 10.000 rials iraniens (600 NF), évitant l'achat des Typha aux habitants de la vallée du Shahur. Le chiffre paraît une surestimation, mais montre l'attachement des villageois à cette culture (cliché 6).

\section{Maraîchage.}

Autour de chaque village, sont cultivés extensivement quelques légumes pour la consommation personnelle des villageois: oignons, navets, carottes, radis, betteraves, melons et pastèques, concombres, aubergines, épinards, et diverses herbes aromatiques. Mais il existe certaines zones, favorisées par la proximité de Dizful ou celle de la grande route, où est pratiqué continuement et intensivement un véritable maraîchage dont les produits sont destinés, soit à la vente en ville, soit même à l'exportation dans le Nord de l'Iran. En plus des légumes cités ci-dessus, on cultive dans ces zones : tomates, piments, ail, et surtout laitues. Dans ces jardins maraîchers, le désir d'obtenir plusieurs récoltes par an, plusieurs années consécutives, oblige à une fumure intensive. Les laitues, cultivées en assolement avec les concombres, sont tout particulièrement fumées. Nous avons repéré dans le secteur quatre zones de cultures de laitues, et trouvé des bulins dans les eaux de drainage de deux d'entre elles. Sur le trajet des drains, sont creusées des mares qui servent au lavage des salades après la récolte et avant le transport au marché. Les débris de légumes restant après ce lavage fournissent aux bulins une abondante nourriture (cliché 8).

\section{Elevage.}

Il existe dans le secteur un important cheptel constitué d'équins (chevaux, ânes, mulets : 20.000 têtes environ), de bovins (bœufs et buffles : 30.000 têtes environ), de chèvres (15.000 têtes environ), et, surtout, de moutons (100.000 têtes environ). Le rôle direct de ces animaux dans l'écologie des bulins nous semble pouvoir se résumer en quatre points: 1) Les animaux sont abreuvés généralement dans les canaux et les buffles s'y baignent souvent. Ils sont un facteur de dégradation du réseau, causant des fuites qui alimentent des mares stagnantes. 2) Leurs excréments enrichissent les eaux en matières organiques favorables à la vie des bulins. 3) Par contre, le piétinement intensif par les troupeaux rend peut-être certains petits marécages impropres à la vie des bulins. 4) Les animaux (buffles en particulier) peuvent transporter des bulins avec la boue qui reste collée à leurs pattes, et offrir à ces mollusques des chances de coloniser assez loin de leur gîte d'origine.

Les zones insuffisamment drainées constituent des pâturages d'été (surtout pour bovins et équins). Nous montrerons plus loin le danger de ces bas-fonds marécageux. Leur suppression nous semble un pas important dans la préven- 
tion de la bilharziose. Mais il faudra peut-être les remplacer, en tant que pâturages, par des cultures fourragères.

\section{Industrie de l'argile.}

L'argile joue un rôle considérable dans la vie des villageois. Elle constitue la matière première des murs et, mélangée de paille, des terrasses. Elle sert à la fabrication de nombreux ustensiles: fours à pain, mangeoires pour les animaux, nids où sont mises à couver les poules, nombreux récipients de formes et capacités variées, en particulier les silos individuels où sont conservées les céréales. Seule, la poterie cuite est achetée en ville. Pour pouvoir disposer d'argile à tout moment, les paysans creusent à proximité immédiate des villages des trous dans lesquels ils amènent, soit l'eau de drainage d'un jardin proche, soit directement (ceci est plus rare) l'eau d'un canal d'irrigation. La lente sédimentation de l'eau dans ces mares permet de trier les particules argileuses, qui se déposent au-dessus des grains de sable plus grossiers. Ces mares sont rapidement envahies par des algues microscopiques, puis par une végétation aquatique. Elles deviennent alors inutilisables en tant que carrières d'argile, mais elles ne sont pas comblées pour autant. D'autres sont seulement creusées un peu plus loin. Toutes ces mares constituent des gîtes à bulins, d'autant plus dangereux que leurs bords servent aisément de cabinets publics. Les excréments sont, en effet, déposés n'importe où autour du village, mais plus volontiers près d'une collection d'eau (canal, mare). Cette proximité facilite la toilette intime selon le mode rituel musulman ; elle apporte aussi aux bulins des matières azotées profitables à leur pullulation, et favorise d'autre part leur infection par les schistosomes (cf. clichés 4 et 6).

\section{Eau pour les usages domestiques et la boisson.}

Il n'y a aucun système d'adduction d'eau dans les villages. Les canaux d'irrigation fournissent non seulement l'eau pour les besoins agricoles, mais aussi l'eau pour les baignades, l'eau pour les besoins domestiques, l'eau de boisson. Les habitants du secteur ne sont pas difficiles au sujet de la qualité de l'eau. Si elle n'est pas salée, elle est bonne. Il est assez surprenant de voir laver du linge dans de l'eau tenant én suspension assez d'argile pour que la main soit invisible à $10 \mathrm{~cm}$. au-dessous de la surface. Pour la boisson, l'eau puisée est généralement placée dans un récipient d'argile poreuse, posé sur un bâti en bois, qui joue le rôle de décanteur en même temps qu'il assure un refroidissement par évaporation.

Pendant les derniers mois de l'hiver, lorsque les canaux sont vidés pour curages et réfections, les villageois utilisent l'eau mise en réserve dans lés mares dont il a été question au paragraphe précédent. L'eau s'évapore lentement à cette époque de l'année, et les pluies la renouvellent plus ou moins. 


\section{Répartition de BULINUS TRUNCATUS}

\section{A. Modes et valeur de la prospection effectuée.}

La prospection du secteur a été effectuée entre le mois de décembre 1959 et le mois de mai 1961. La recherche des bulins a partout été faite au moins deux fois, une fois en saison chaude et sèche (mai à septembre), une fois en saison fraîche et pluvieuse (novembre à mars). La prospection a été facilitée par la planéité générale du pays, par la rareté ou l'absence des arbres, par l'existence de monticules isolés (tells) qui, de loin en loin, dominent la plaine et offrent des vues panoramiques assez étendues. En revanche, la prospection a été compliquée par la rareté des ponts ou des gués aménagés permettant la traversée des cours d'eau et des grands canaux. La terrible chaleur de l'été a aussi été un obstacle.

Nous nous sommes attaché à mettre en évidence, non seulement les gîtes hébergeant actuellement des bulins, mais aussi les gîtes anciens contenant seulement des tests subfossilisés.

Dans les collections d'eau contenant une végétation macroscopique, type de gîte de longtemps le plus fréquent, la recherche des mollusques a été faite avec le filet plat, déjà utilisé par l'Institut de Parasitologie de Téhéran (1). Ce filet, robuste et relativement léger, permet de bien racler le fond, et de laver facilement la boue éventuellement ramenée. Au cours de plusieurs vérifications, le nombre de bulins ramenés par un coup de filet s'est montré égal au 1/5 du nombre de bulins existant sur $1 \mathrm{~m}^{2}$ de surface de gîte. Le défaut de ces filets est la difficulté de les bien laver après usage. Plusieurs fois, de petits bulins sont restés pris entre la toile métallique et le cadre. Retrouvés ensuite lors de l'exploration d'une autre collection d'eau, ils ont créé des confusions.

Dans les collections d'eau sans végétation, l'examen de la face inférieure des pierres immergées s'est montré le meilleur moyen de trouver les mollusques et leurs pontes.

Dans les mares desséchées, le lavage d'échantillons de terre $(20 \times 20 \mathrm{~cm}$. $\times$ $10 \mathrm{~cm}$. de hauteur), prélevés au creux le plus profond, nous a donné d'intéressants résultats. Sur 20 gîtes découverts dans des collections d'eau asséchées, 15 l'ont été par l'examen de la surface du sol, 5 par lavage de la terre prélevée après examen négatif de la surface.

L'examen des boues de curage mises à sécher sur les bords des canaux a été un mode de prospection très fructueux : 20 gîtes ont été découverts ainsi.

(1) Cadre métallique de $35 \times 35 \mathrm{~cm}$., le bord antérieur convexe vers l'avant, tendu d'une toile métallique à fils écartés de $1 \mathrm{~mm}$. 5 , et emmanché par un tube métallique de $12 \mathrm{~cm}$. sur une tige de bois de $140 \mathrm{~cm}$. de long. Poids $2 \mathrm{~kg}$.

ANN. DE PARASITOLOGIE, T. XXXVII, $x^{\circ} 3 .-1962$ 


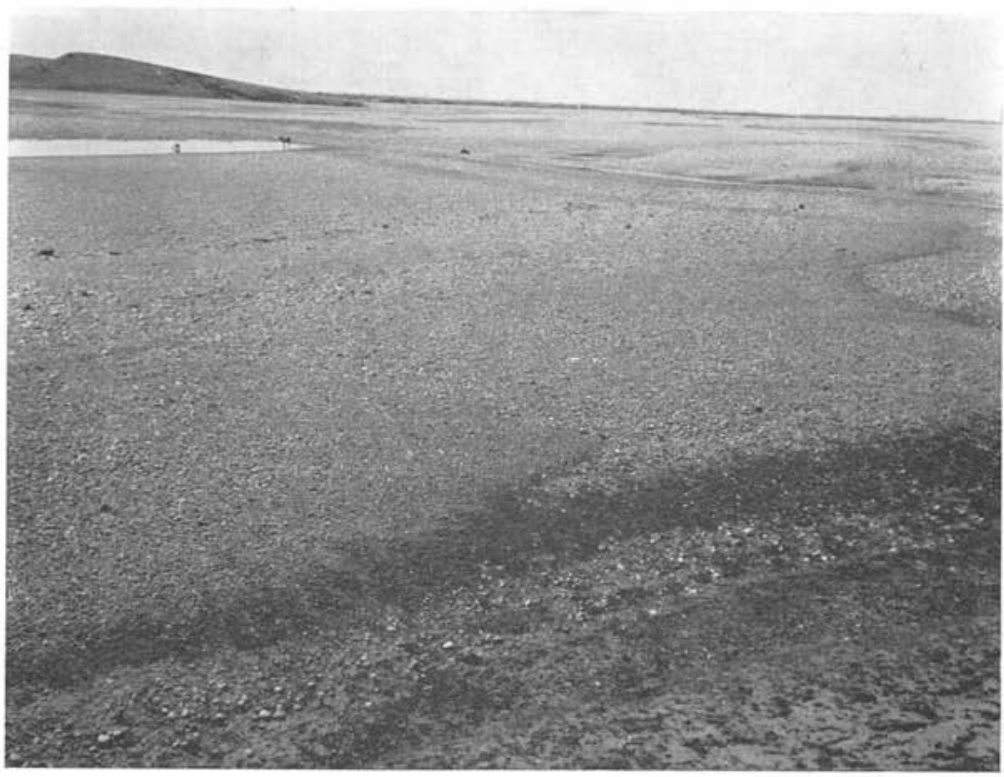

Fig. 1. - Plaine d'inondation de Kahunak. Faciès à galets

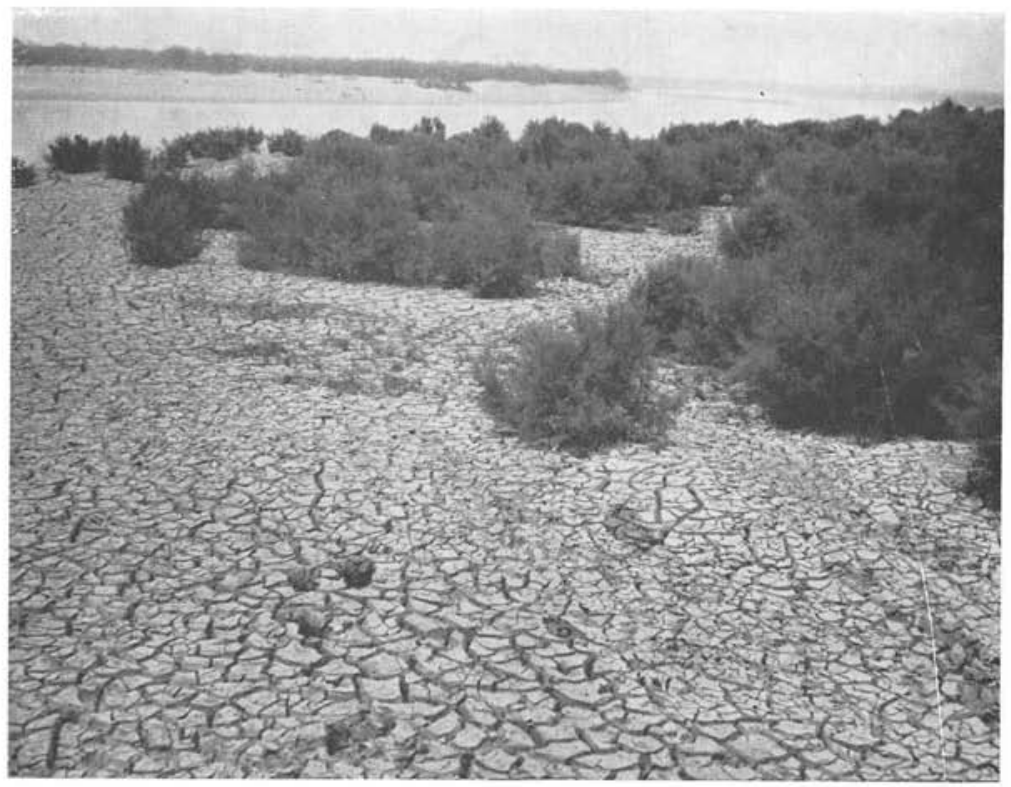

Fıg. 2. - Plaine d'inondation du Karkheh. Faciès à tamarix 


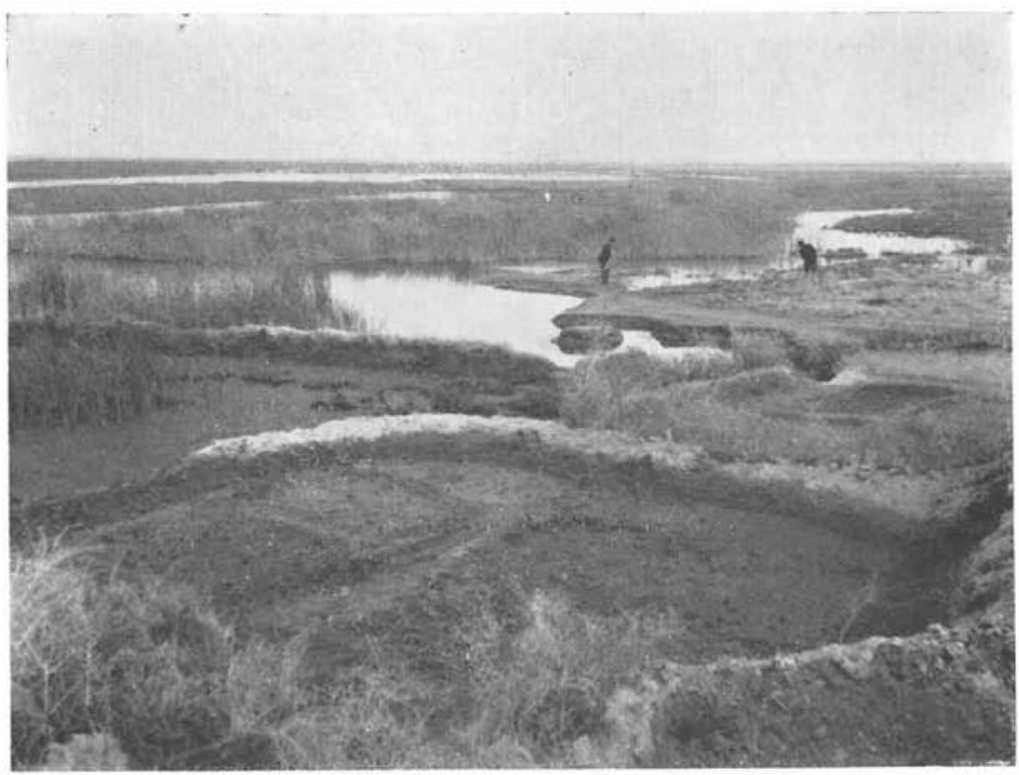

Fig. 3. - Plaine d'inondation du Shahur. Faciès marécageux

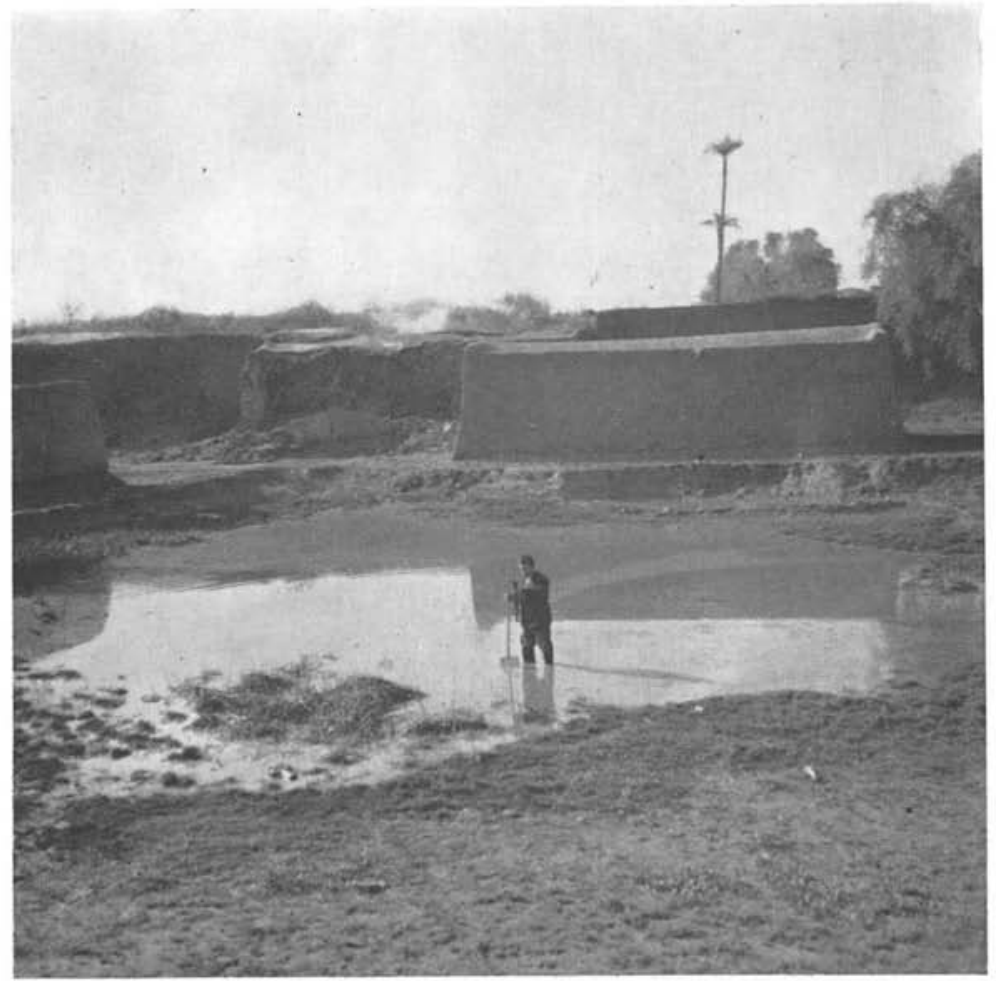

Fig. 4. - Chagha Sorkh. Mare artificielle d'emprunt de terre. Stade jeune (Gite $\mathrm{n}^{\circ} 27 a$ ) 


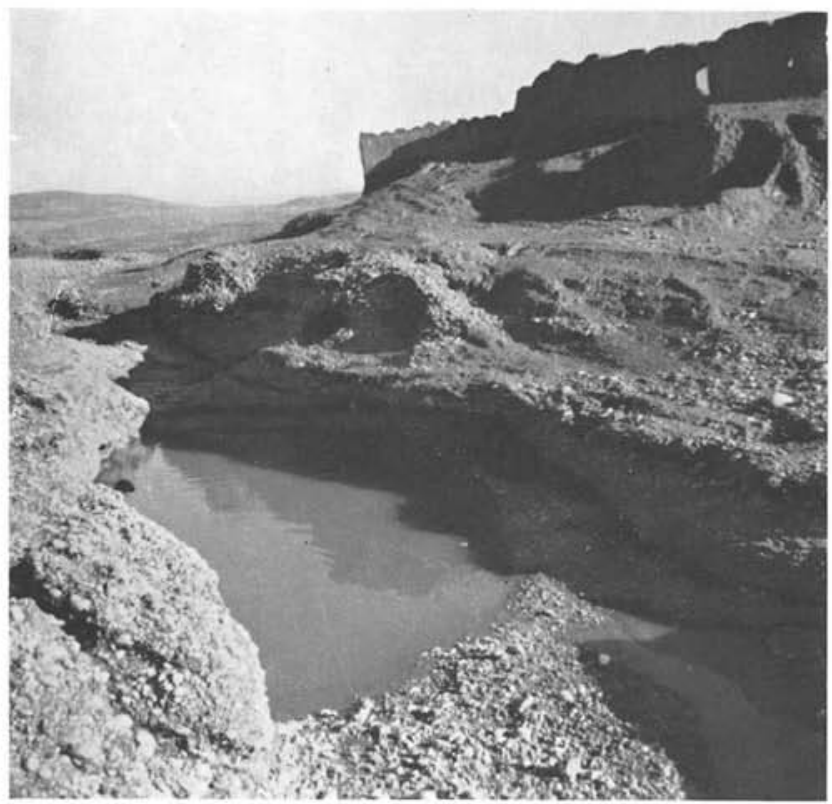

FIG. 5. - Gîte sans végétation macroscopique. Ab Koveit (Gîte $\mathrm{n}^{\circ} 31$ )

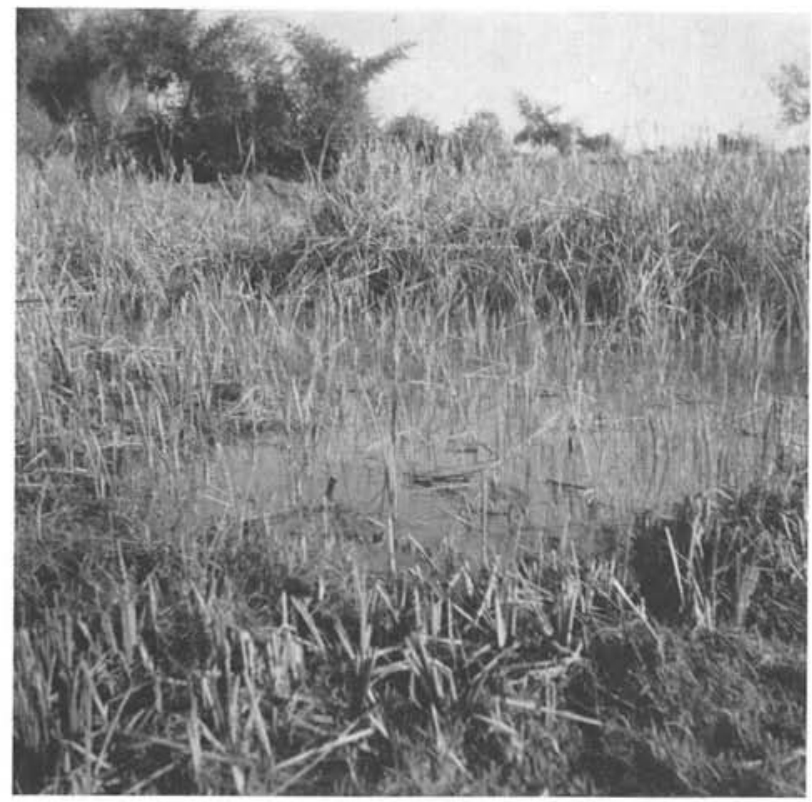

Fig. 6. - Shahabad. Mare d'emprunt de terre avec plantation de Typha (Gîte $\mathrm{n}^{\circ} 24 b$ ) 


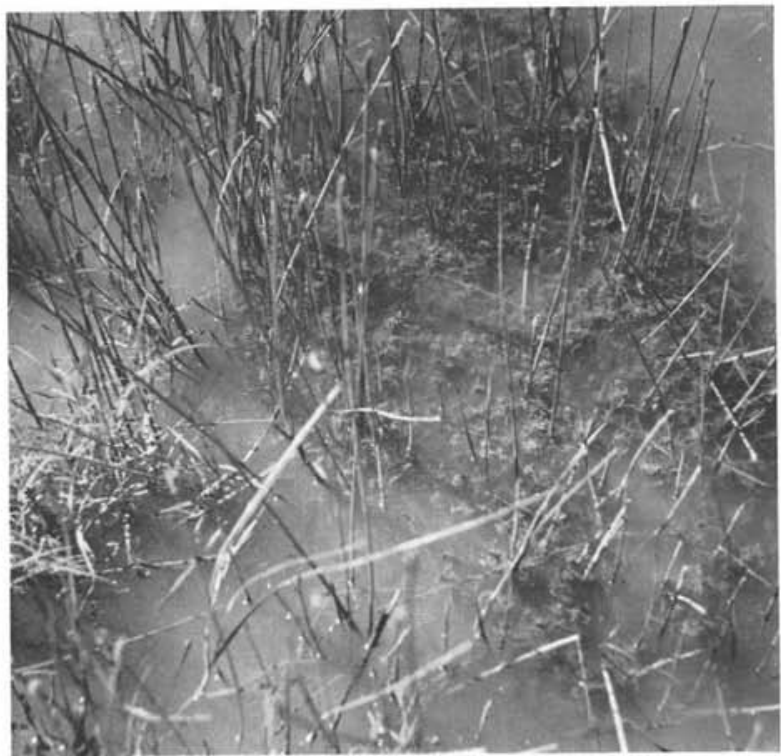

Fig. 7. - Bonvar Hoceïne. Feutrage de Characées (Gîte $\mathrm{n}^{\circ} 8 b$ )

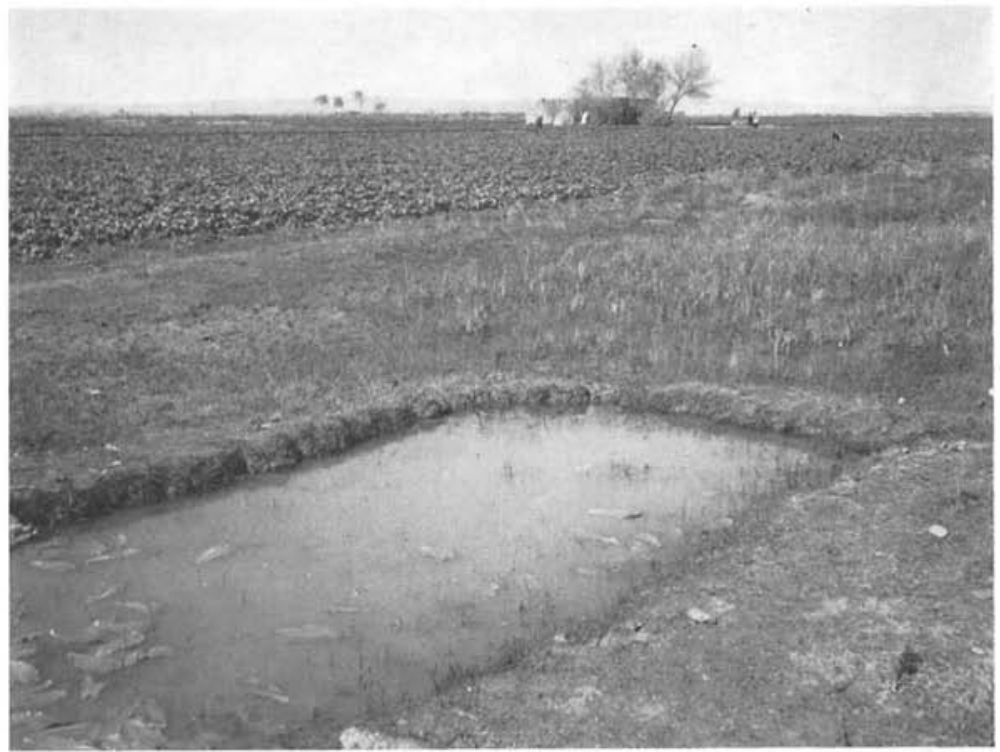

FIG. 8. - Ambarieh. Mare pour lavage des laitues (Gîte $\mathrm{n}^{\circ} 1^{1}$ 


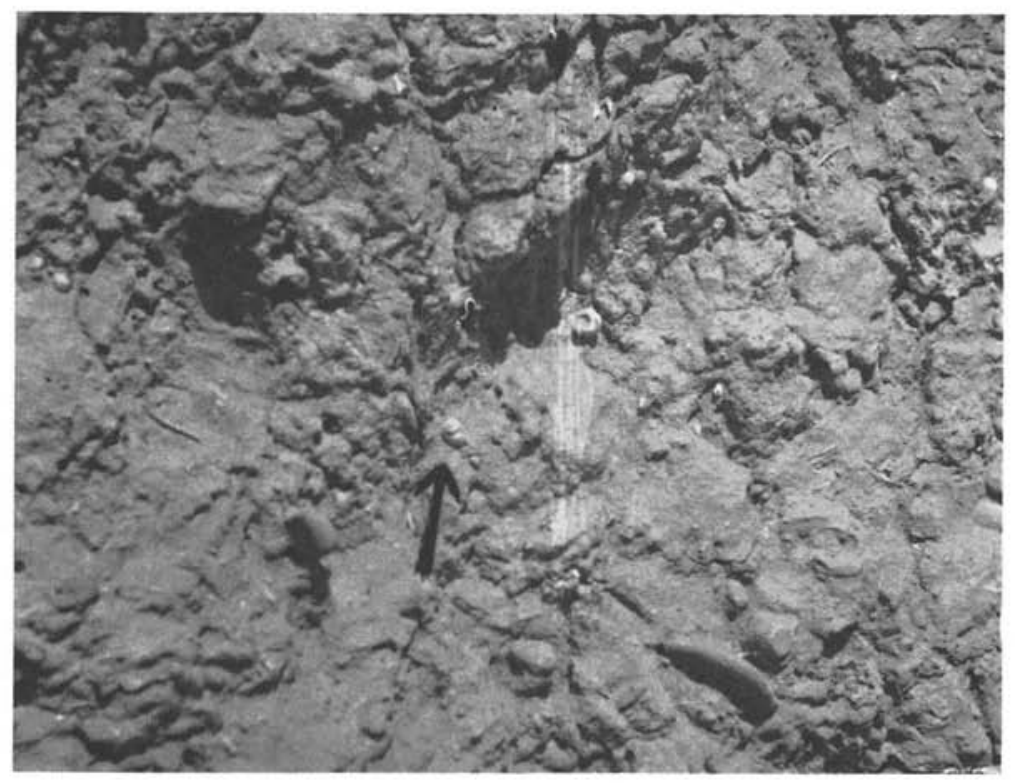

Fig. 9. - Bulin fossile dans un mur d'argile

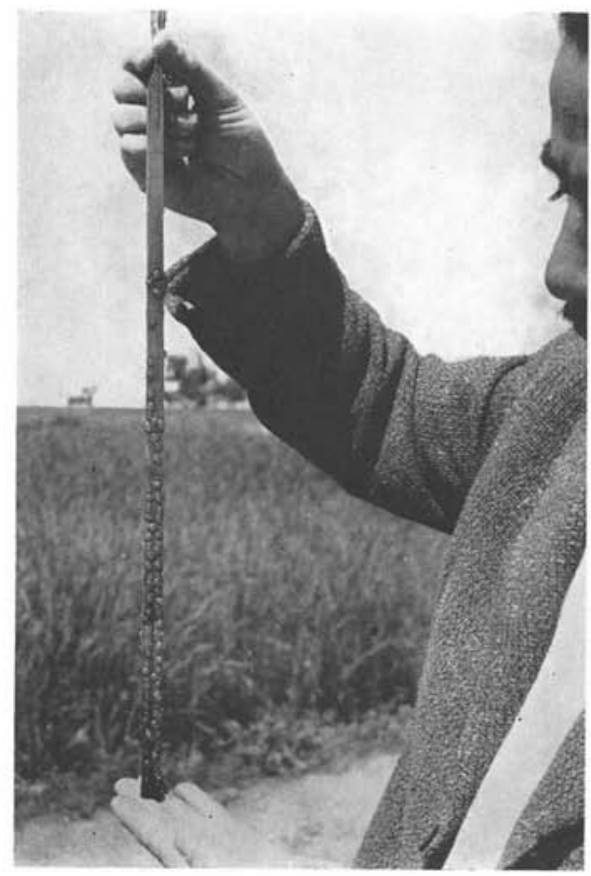

FIG. 10. - Pontes de Bulins sur une feuille flottante de Typha. Q. Seyed (Gîte $\mathrm{n}^{\circ}$ 13) 
Enfin, l'examen systématique des murs d'enceinte des villages et des jardins nous a très souvent permis de trouver des tests de bulins mis à jour par lès lavages des pluies. Ce procédé de prospection, très rapide, nous a cinq fois amené à trouver un gîte qui nous aurait sans cela échappé (oliché 9).

Il n'a pas été fait un usage systématique des pièges, méthode coûteuse en temps et en essence dès qu'il s'agit de zones éloignées de la base. Quelques expériences ont montré que des planchettés immergees pendant trois à six jours pourraient constituer d'excellents pièges (2). Mais le meilleur piège s'est révélé être la plaquette de porcelaine support de nos thermomètres maximum-minimum. Le premier indice de la repopulation du gîte $\mathrm{n}^{\circ} 8 a$ (Bonvar-Hoceine) a été une ponte déposée sur cette plaque.

Au total, la prospection réalisée n'a pas été parfaitement homogène et n'est vraisemblablement pas exhaustive. D'une part, les $80 \mathrm{~km}^{2}$ très difficilement accessibles de la plaine d'inondation du Diz sont restés insuffisamment prospectés. D'autre part, les risques d'avoir méconnu un gîte sont certainement plus grands pour la partie Sud du secteur qu'ils ne le sont pour la partie Nord, plus proche de la base de Dizful, et pourvue d'un réseau routier plus dense. Enfin, les prospecteurs ont toujours porté plus d'attention aux environs immédiats des villages qu'aux espaces inhabités séparant ceux-ci.

Telle que, cette prospection a permis de dénombrer 144 gîtes de B. truncatus. Nous entendons par gîte toute collection d'eau, de surface définie, contenant ou ayant contenu des bulins. Ces gîtes se groupent assez naturellement en 102 stations. Nous employons ce terme de station pour désigner toute aire de petite surface (pas supérieure à $1 \mathrm{~km}^{2}$ en principe), sur laquelle existent ou ont visiblement existé un ou plusieurs gîtes de bulins. Cette notion de « station » est généralement très valable dans le secteur étudié, car chaque station est séparée de ses voisines par de larges intervalles vierges de bulins. Lorsque, exceptionnellement, nous avons eu affaire à une juxtaposition de gîtes sur une longue étendue (cas du canal Nahr-e-Bahman — cf. p. 258 - ou du cours inférieur du Shahur), nous avons arbitrairement divisé cet ensemble en stations, chaque section de $2 \mathrm{~km}$. de long formant une station. Les résultats de la prospection sont schématisés par les cartes 3 et 4 .

\section{B. Distribution de B. TRUNCATUS dans le secteur étudié.}

\section{Homogénéité.}

Si l'on considère la répartition des stations de $B$. truncatus sur l'ensemble de la surface du secteur, la première impression est celle d'une relative homogénéité. La moitié Est du secteur compte 47 stations, alors que la moitié Ouest en compte 55. La moitié Nord du secteur compte 44 stations, alors que la

(2) Malheureusement trop tentants pour les villageois dans un pays cù la bois est rare. 
Tableau III. - RÉPARTITION DES STATIONS EN FONCTION DE L'ORIGINE DES EAUX CRÉANT LES GîTES

\begin{tabular}{|c|c|c|c|c|}
\hline Zones & 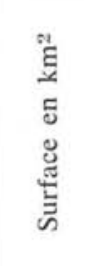 & 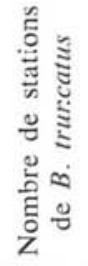 & 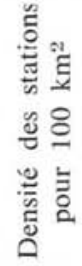 & Observations \\
\hline $\begin{array}{l}\text { Zones non irriguées: } \\
\text { Nord et Est du secteur. } \\
\text { Sud du secteur ........ }\end{array}$ & 175 & 0 & - & $\begin{array}{l}\ldots . \text { Plaines; les gîtes } \\
\text { sont de petites } \\
\text { sources } \\
\ldots . \text { Collines arides }\end{array}$ \\
\hline Total zones non irriguées. & 351 & 3 & 0,8 & \\
\hline $\begin{array}{l}\text { Lits des cours d'eau et } \\
\text { plaines d'inondation: } \\
\text { du Karkheh } . . . \ldots \ldots\end{array}$ & 27 & 1 & 一 & $\begin{array}{l}\text {... En majorité bois } \\
\text { de tamaris }\end{array}$ \\
\hline du Bala Rud $\ldots \ldots \ldots$ & 6 & 0 & 一 & $\cdots$ Plaines de graviers \\
\hline du Lureh et Shureh ... & 38 & 0 & 一 & ... Plaines de graviers \\
\hline du Diz $\ldots \ldots \ldots \ldots$ & 118 & 2 & 一 & $\begin{array}{l}\text { Faciès variés, pros- } \\
\text { pection difficile }\end{array}$ \\
\hline du Shahur...$\ldots \ldots$. & 6 & 8 & 一 & $\begin{array}{l}\text { … Marécages à végé- } \\
\text { tation basse }\end{array}$ \\
\hline Total zones innondées ... & 195 & 11 & 5,6 & \\
\hline $\begin{array}{l}\text { Zones irriguées: } \\
\text { par les eaux du Karkheh }\end{array}$ & 325 & 28 & 8,6 & \\
\hline $\begin{array}{l}\text { par les eaux du Diz (ca- } \\
\text { naux) } \ldots \ldots \ldots \ldots \ldots \ldots \ldots\end{array}$ & 436 & 32 & 7,3 & \\
\hline $\begin{array}{l}\text { par les eaux du Diz } \\
\text { (pompages) } \ldots \ldots \ldots \ldots \ldots\end{array}$ & 87 & 4 & 4,6 & $\begin{array}{l}\text { Essentiellement : } \\
\text { plantations de can- } \\
\text { nes à sucre }\end{array}$ \\
\hline par les eaux du Shahur. & 129 & 12 & 9,2 & $\begin{array}{l}\text {... Beaucoup de gîtes } \\
\text { dans les canaux } \\
\text { d'irrigation }\end{array}$ \\
\hline par les eaux du Lureh. & 124 & 12 & 9,7 & \\
\hline Total zones irriguées .... & 1.101 & 88 & 8 & \\
\hline Total général ...... & 1.647 & 102 & 6,2 & \\
\hline
\end{tabular}


moitié Sud en compte 58. Mais cette impression d'homogénéité repose sans doute sur un biais dans la collection des données de base. La moitié Nord du secteur, nous l'avons dit plus haut, a probablement été mieux prospectée que la moitié Sud.

Tableau IV. - RÉPARTITION DES STATIONS PAR BASSINS DRAINANTS

\begin{tabular}{|c|c|c|c|c|c|c|}
\hline \multirow[b]{2}{*}{ Bassins } & \multicolumn{3}{|c|}{ Surfaces en $\mathrm{km}^{2}$} & \multicolumn{3}{|c|}{ Stations de $B$. truncatus } \\
\hline & 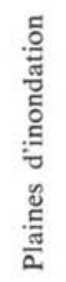 & $\begin{array}{l}\tilde{E} \\
\text { ¿d } \\
\tilde{E} \\
\tilde{\delta} \\
\tilde{D}\end{array}$ & 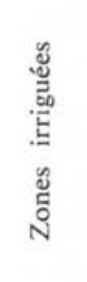 & 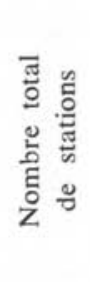 & 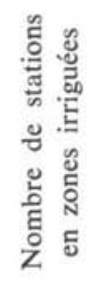 & 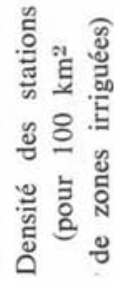 \\
\hline BALA RUD & 6 & 38 & 75 & 3 & 3 & 4 \\
\hline DIZ & 118 & 45 & 304 & 24 & 22 & 7,2 \\
\hline KARKHEH & 27 & 0 & 38 & 6 & 5 & 13,2 \\
\hline SHAHUR & 6 & 133 & 342 & 39 & 31 & 9,1 \\
\hline LUREH & 38 & 135 & 165 & 19 & 16 & 9,7 \\
\hline AB JIROB & 0 & 0 & 177 & 11 & 11 & 6,2 \\
\hline TOTAL & 195 & 351 & 1.101 & 102 & 88 & 8 \\
\hline
\end{tabular}

Un examen plus détaillé montre la concentration des stations en certaines zones, certaines autres, en contraste, étant pratiquement indemnes. L'explication de ces « taches blanches » est quelquefois apparente: les plaines d'inondation recouvertes de galets, ou bien les zones rigoureusement sèchés, constituent de vastes surfaces où les bulins ne peuvent s'implanter; la * Sugar cane area » a été l'objet d'un tel bouleversement de terrain qu'il est normal de n'y pouvoir déceler actuellement ni bulins vivants, ni traces de bulins subfossiles. Parfois, en revanche, la cause de l'absence des bulins nous échappe. Nous n'avons pu mettre en évidence aucune différence entre la zone indemne qui occupe le centre de la zone pilote d'irrigations et les zones infestées qui s'étendent immédiatement au Nord-Est et au Sud. 


\section{Gîtes naturels et gîtes artificiels.}

Le nombre des gîtes naturels trouvés dans le secteur est relativement faible : moins du dixième du total des gîtes recensés. Ces gîtes naturels sont de deux sortes: 1) de petites sources ou résurgences (gîtes $\mathrm{n}^{\circ s} 25,26$ et 31) ; 2) des marécages dans le lit ou la plaine d'inondation de rivières ou grands drains naturels (gîtes $\mathrm{n}^{\circ *} 110,112,117,118,119,120,122 a, 130,131,132$ ). On remarquera que tous les gîtes du premier type se trouvent dans la partie Nord du secteur, et que tous ceux du second type se trouvent dans la partie Sud.

Tous les autres gîtes ont un caractère plus ou moins artificiel. Ce caractère est incontestable lorsqu'il s'agit de petites mares créées de main d'homme, type de gîte fréquent dans la moitié Nord-Est du secteur, et notamment dans la zone pilote d'irrigation. Il est incontestable également lorsqu'il s'agit de canaux d'irrigation, type de gîte relativement rare, rencontré essentiellement en bordure Sud-Ouest du secteur. Le caractère artificiel est moins net pour un grand nombre d'autres gîtes que nous ne pouvons cependant pas considérer comme naturels, pour l'une ou l'autre des deux raisons, jouant souvent simultanément : 1) Il s'agit d'eaux stagnantes parce qu'arrêtées par une barrière artificielle. Cette barrière peut être la levée de terre de la voie ferrée, ou d'une route, ou celle d'un canal. Ce dernièr cas est fréquent, qu'il s'agisse de canaux actuellement en usage, ou qu'il s'agisse d'anciens canaux abandonnés. 2) L'eau qui alimente le gîte pendant la plus grande partie de l'année provient plus ou moins directement du réseau d'irrigation (fuites, débordements, eaux d'irrigation en excès, drainage des cultures).

\section{Répartition des bulins et réseau hydrographique.}

Il paraît intéressant de savoir s'il existe une relation entre la répartition des stations de bulins et l'origine de l'eau d'irrigation, ce qui suggérerait, soit une influence du chimisme de l'eau, soit une infestation d'amont en aval dans le sens du courant de l'eau; ou bien une relation entre la répartition de ces stations et les différents bassins drainants, ce qui suggérerait une infestation d'aval en amont, en sens inverse du courant de l'eau. Pour cela, nous considérerons seulement les gîtes situés dans les zones irriguées. Les plaines d'inondation des rivières, en effet, comme les zones sèchès, présentent des faciès divers et difficilement comparables (cf. p. 237). Au contraire, les zones irriguées, qui contiennent les 9/10 du total des gîtes, constituent un ensemble assez homogène. Tous les grands propriétaires ordonnent à peu près les mêmes cultures, et la proportion de terres réservées à chaque culture varie peu d'une propriété à l'autre.

Le tableau III et la carte 3 montrent qu'il est difficile d'établir une relation entre la distribution des bulins et l'origine des eaux irriguant les diverses parties du secteur. Les différences observées ne sont pas statistiquement signifi- 
catives. A plus petite échelle, il est difficile de parlèr de prédilection pour aucun canal primaire donné. Il existe une cinquantaine de canaux primaires dans le secteur. Sur les réseaux issus de ces canaux, 17 seulement sont indemnes, la plupart de ceux-ci couvrant une très faible surface. Des 10 réseaux d'irrigation d'une surface supérieure à $30 \mathrm{~km}^{2}$, deux seulement comportent plus de quatre stations : celui du canal Shamoun, qui en compte sept, et celui du canal Harmushi, qui en compte 14; mais ce dernier réseau est immense et couvre près de $200 \mathrm{~km}^{2}$, soit le $1 / 5$ de la surface totale des terres irriguées du secteur.

Le tableau IV et la carte 4 montrent qu'il est également difficile d'établir une relation entre distribution des bulins et bassins drainants. Là encore, les différences observées ne sont pas significatives.

\section{Distribution des bulins et physiologie de l'irrigation.}

Si la notion « d'unité anatomique » au sein du réseau des canaux éclaire mal ce que nous savons de la répartition des bulins, il n'en va pas de même pour la notion « d'élément fonctionnel » mis en jeu par l'irrigation. Dans cette optique, en comparant - un peu arbitrairement sans doute — irrigation et circulation sanguine, on peut distinguer dans un réseau d'irrigation :

1) un système artériel (afférent), constitué par les canaux d'irrigation primaires, secondaires, tertiaires, etc... ;

2) un système capillaire: les champs irrigués ;

3) un système veineux (efférent), constitué par les drains ;

4) des systèmes « porte », c'est-à-dire des drains employés secondairement comme canaux d'irrigation.

Si nous examinons la répartition des gîtes de bulins dans ces diverses parties fonctionnelles, telle qu'elle se présente dans le secteur, nous notons que :

Le \& système artériel » [1] proprement dit offre exceptionnellement aux bulins des conditions favorables. Si nous exceptons le cas très particulier du canal Nahr-e-Bahman, analysé plus loin, 1/6 seulement des gîtes reconnus (24/144) concernent des canaux d'irrigation. Dans la moitié des cas, il n'a pas été trouvé de bulins vivants, mais seulement des tests dans les boues de curage. Enfin, les gîtes 114 et 115 exceptés (3), les bulins vivants trouvés étaient toujours rares et ont disparu par la suite rapidement.

Les champs irrigués [2] constituent très exceptionnellement des gîtes à bulins, à l'exception de quelques rizières (gîtes $40 a, 44 b$ ). Encore doit-on noter dans

(3) Ces deux gîtes sont actuellement vides de bulins, après en avoir hébergé en abondance pendant deux années consécutives. 
ces cas que les bulins se trouvent seulement dans la partie « aval » de la rizière, du côté « veine » plutôt que du côté « artère ». On remarque aussi qu'il existe un gîte autre que la rizière, non loin de celle-ci, dans la même station.

C'est au niveau des drains [3], et particulièrement au niveau de l'origine de ceux-ci (lacs ou plexus veineux, serions-nous tentés de dire), que se constituent le plus grand nombre de gîtes liés à l'irrigation (gîtes 1, 2, 3, 4b, 5a, 6, 7, $8 a, b, c$, etc...). Tout obstacle au drainage des eaux de surface - levées de terre des routes, voies ferrées et canaux, dépressions naturelles, mares d'emprunts de terre - est l'origine de gîtes à bulins, pour peu que les eaux mal drainées stagnent avec une certaine pérennité (4). Les petits drains, à faible débit, à cours mal défini dans un lit large, mais peu profond, constituent également des gîtes à bulins. Plus en aval, les grands drains se comportent comme des rivières et n'offrent plus qu'exceptionnellement des gîtes favorables aux bulins.

Les « systèmes porte »[4] constituent, comme les drains qu'ils sont effectivement à l'origine, des gîtes à bulins. De plus, ils offrent à ces derniers des possibilités de dispersion et de colonisation remarquables. Le cas du canal Nahr-e-Bahman, « veine porte » la plus notoire du secteur (gîtes $\mathrm{n}^{\circ s} 9 b, 11 b$, $12,53)$, est typique à cet égard. Ce canal a son origine dans le Diz, au NordEst de Dizful. Après un trajet N.-N.O./S.-S.E. de $15 \mathrm{~km}$. environ, au cours duquel il ne donne aucune branche secondaire, le Nahr-e-Bahman se jette dans la branche Nord-Est du canal Dahli. Le Nahr-e-Bahman est exceptionnellement utilisé par son propriétaire, dont les terres se trouvent à ShalghehiOlia, à $20 \mathrm{~km}$. au Sud-Est de Dizful, et seulement lorsque les eaux du réseau Dahli sont insuffisantes. (En fait, nous avons vu ce canal ouvert en tête trois fois seulement au cours de l'année 1960). Pendant ces longues interruptions de service à titre « artériel », le Nahr-e-Bahman ne s'assèche pas complètement. Il recueille les eaux de drainage de différents autres réseaux, et, tout particulièrement, les eaux de drainage du maraîchage pratiqué à l'Est de Dizful avec les eaux du canal Nahr-e-Hajian. Sur une longueur de $8 \mathrm{~km}$., l'eau stagne dans le Nahr-e-Bahman. Des Characées et des Ceratophyllum s'y développent, et nous avons pu compter, au mois d'août 1960, dans ce canal-drain, jusqu'à 1.000 bulins par mètre linéaire! Chaque mise en charge du canal, que ce soit par ouverture en tête, comme nous l'avons vu en septembre, ou par pluie abondante, comme nous l'avons vu en novembre, fait un massacre de ces bulins, mais en distribue aussi, pour colonisation éventuelle, sur les $50 \mathrm{~km}^{2} \mathrm{du}$ réseau Dahli Nord-Est.

(4) Cette pérennité est évidemment une condition essentielle. Sa nécessité explique pourquoi les cultures n'ont pas toutes la même importance comme facteur d'entretien des populations de mollusques. Les eaux en excès auront d'autant plus de chances de créer des gîtes à bulins qu'elles proviendront de cultures occupant le sol toute l'année pendant plusieurs années consécutives (jardins, maraîchages), ou de cultures irriguées en été assez abondamment et assez longtemps pour que les pluies viennent à temps sauver les mollusques de la dessiccation (rizières). 


\section{Evolution des populations de B. TRUNCATUS}

\section{A. Conditions d'observation.}

Nous n'avons pas pu observer tout au long de l'année les populations de B. truncatus vivant dans les grands marécages (stations $\mathrm{n}^{\circ \mathrm{s}} 117$ à 120 , et 130 à 132). Nous pouvons seulement dire que la densité de ces populations était extrêmement faible en hiver, au printemps et en été. La situation ne doit pas être très différente en automne. Dans ces grandes collections naturelles d'eau permanente, les conditions écologiques restent stables ; un équilibre biologique s'est établi et se maintient depuis longtemps, laissant peu de chances pour les brusques progressions ou régressions qui s'observent, au contraire, dans les petites collections d'eau temporaires ou à régime très irrégulier. C'est sur ces dernières (l'immense majorité des gîtes trouvés dans le secteur étudié) que nous avons essayé d'obtenir un maximum de renseignements.

D'une enquête longitudinale poursuivie par examens mensuels sur dix gîtes choisis aussi différents que possible les uns des autres, et de la comparaison entre les renseignements ainsi recueillis et les observations faites sur des élevages au laboratoire, nous espérions tirer des conclusions sur les époques de multiplication des bulins et les conditions favorables à leur développement. De ces conclusions, nous pensions pouvoir déduire ensuite quels procédés de destruction choisir, et à quel moment de l'année les appliquer. En fait, les résultats ont été décevants.

Tout d'abord, les élevages au laboratoire n'ont pas donné ce qu'on pouvait en attendre. Les deux premières générations ont paru souffrir des conditions artificielles où elles étaient placées. En dépit de l'apparente constance de ces conditions, l'activité reproductrice des bulins a été irrégulière, relativement faible, et, paradoxalement, en discordance complète avec celle des bulins des gîtes d'où provenaient les souches. Les générations ultérieures paraissaient mieux acclimatées, mais l'observation sur celles-ci a été malheureusement trop courte.

En second lieu, le rythme d'examen mensuel s'est révélé insuffisant pour saisir toutes les brusques modifications des conditions écologiques et leurs répercussions sur les populations de bulins.

Enfin, les variations biologiques n'ont été les mêmes dans aucun des dix gîtes observés. Ainsi, le maximum de la densité des bulins a été atteint en avril dans les gîtes $\mathrm{n}^{\circ \mathrm{s}} 8 a$ et 15 , en mai dans le gîte $\mathrm{n}^{\circ} 20 e$, en juin dans les gîtes $\mathrm{n}^{\circ \mathrm{s}} 8 a$ et 13 , en juillet dans le gîte $\mathrm{n}^{\circ} 19 b$, en août dans les gîtes $\mathrm{n}^{\circ \times} 1$ et 10 , en octobre dans les gîtes $\mathrm{n}^{\circ \Sigma} 27 a$ et 31 , en décembre dans le gîte $\mathrm{n}^{\circ} 24 a$. Ces maxima varient, par ailleurs, d'un bulin pour $2 \mathrm{~m}^{2}$ à 1.500 bulins au m² (tableau V).

Ann. de Parasitologie, T. XXXVII, N ${ }^{\circ} 3 .-1962$. 


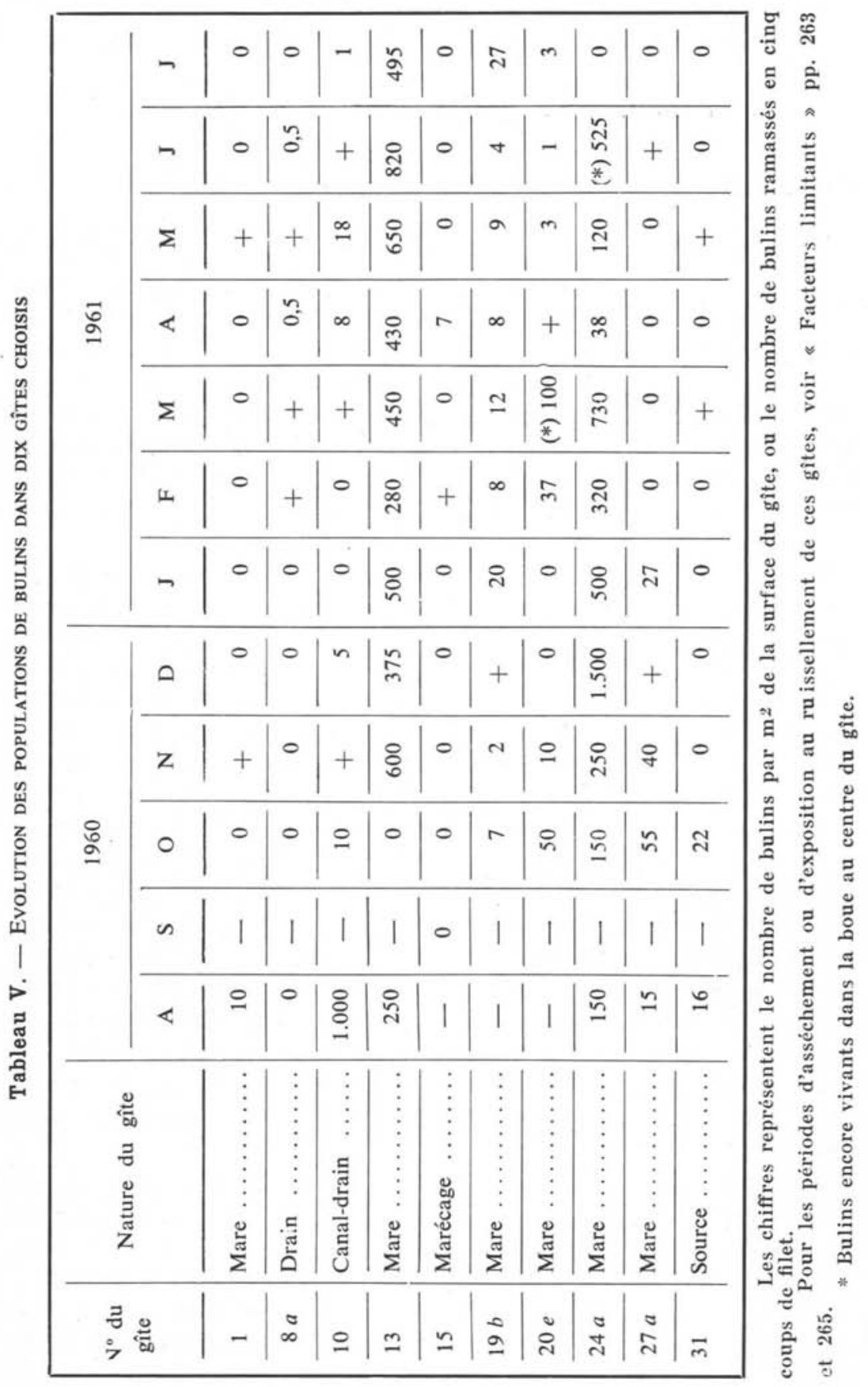


Le caractère * individuel » de ces variations décourage les essais de généralisation.

Cependant, d'intéressantes observations ont pu être faites.

\section{B. Rapidité de développement des populations}

\section{de B. TRUNCATUS}

Dans les élevages au laboratoire, si l'activité reproductrice a été irrégulière, elle a par moments atteint une cadence étonnamment rapide. En novembre 1960 et en mai 1961, nous avons vu le nombre moyen des pontes dépasser un par individu et par jour. En juin 1961, nous avons observé des temps d'incubation de sept, et même de six jours. Au total, nous avons eu en mai 1961 les premiers arrière-petits-enfants ( $4^{\circ}$ génération) des bulins pris dans la nature en août 1960 .

Dans les petites collections d'eau stagnante, le développement des populations de bulins semble aussi pouvoir être très rapide, et, dans certaines conditions, presque explosif.

Dans le gîte $\mathrm{n}^{\circ} 24 a$ (Shahabad), on pêche en novembre 50 bulins par coup de filet, dont $20 \%$ de taille inférieure à $3 \mathrm{~mm}$. ; en décembre, 300 bulins par coup de filet, dont $90 \%$ de taille inférieure à $3 \mathrm{~mm}$. Le gîte $\mathrm{n}^{\circ} 13$ (Q. Seyed) a été asséché pendant plus d'un mois en septembre-octobre 1960. Le 12 novembre, dans le gîte remis en eau depuis un mois environ (déclaration des habitants), chaque coup de filet ramenait une vingtaine de bulins, presque tous de grande taille. Le 19 novembre, un coup de filet en ramenait 120, dont $66 \%$ de taille inférieure à $3 \mathrm{~mm}$.

Dans les deux cas, ce développement explosif a été suivi d'une réduction relative. En janvier, on pêchait dans le gîte $\mathrm{n}^{\circ} 13$, comme dans le gîte $\mathrm{n}^{\circ} 24 a$, une centaine de bulins par coup de filet. Nous ne pouvons dire si cette réduction de densité qui, dans les deux cas, s'est produite au même moment de l'année, est due aux effets d'une surpopulation, ou bien correspond à un ralentissement saisonnier de l'activité reproductrice. Chacun de ces phénomènes joue un rôle certain dans la biologie de B. truncatus au Khouzistan.

Le rôle de la surpopulation apparaît nettement au cours de l'évolution ultérieure de la population de bulins du gîte $\mathrm{n}^{\circ} 13$, évolution schématisée dans le tableau VI. Entre mars et juin 1961, cette population n'a pas doublé malgré une activité reproductrice extraordinaire. Nous avons pu dénombrer, en avril, 68 pontes de bulins sur une seule feuille de typha arrachée et flottant sur l'eau (cliché 10).

Par ailleurs, l'activité reproductrice montre des variations saisonnières.

Ces variations portent relativement peu sur la ponte elle-même. Celle-ci paraît plus abondante en automne et à la fin du printemps qu'en juillet ou en décembre. Mais, pendant la période d'observation 1960-1961, des pontes fraî- 
ches ont été recueillies tout au long de l'année. Il n'y a pas eu de diapause hivernale, notamment, comme cela s'observe en Afrique du Nord. Au momen le plus froid de l'année, le nombre moyen d'œufs par ponte était encore élevé : 14 en janvier, 12 en février.

Tableau VI. Evolution DE LA STRUCTURE DE LA POPULATION DE BULINS A Q. SEyed d'octobre 1960 a JUILlet 1961

\begin{tabular}{|c|c|c|c|c|c|c|c|c|}
\hline \multirow{2}{*}{. Mois } & \multirow{2}{*}{ 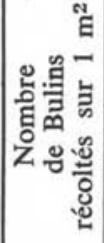 } & \multicolumn{7}{|c|}{ Répartition par tailles des bulins récoltées } \\
\hline & & $\begin{array}{l}2-3 \\
\mathrm{~mm} .\end{array}$ & $\begin{array}{c}4-5 \\
\mathrm{~mm} .\end{array}$ & $\begin{array}{c}6-7 \\
\mathrm{~mm} .\end{array}$ & $\begin{array}{c}8-9 \\
\text { mm. }\end{array}$ & $\begin{array}{c}10-11 \\
\mathrm{~mm} .\end{array}$ & $\begin{array}{c}12-13 \\
\mathrm{~mm} .\end{array}$ & $\begin{array}{c}14-15 \\
\mathrm{~mm} .\end{array}$ \\
\hline Octobre $\ldots$. & 0 & . & .... & assèche & ent du & îte & & . \\
\hline Novembre ... & 600 & 395 & 14 & 12 & 66 & 99 & 14 & 0 \\
\hline Décembre .. & 385 & 215 & 32 & 10 & 7 & 117 & 3 & 0 \\
\hline Janvier ..... & 501 & 36 & 311 & 41 & 39 & 73 & 1 & 0 \\
\hline Février ..... & 283 & 25 & 122 & 37 & 22 & 77 & 0 & 0 \\
\hline Mars $\ldots . .$. & 440 & 54 & 152 & 168 & 37 & 26 & 3 & 0 \\
\hline Avril $\ldots \ldots$ & 432 & 3 & 20 & 279 & 103 & 14 & 13 & 0 \\
\hline Mai $\ldots \ldots$. & 644 & 368 & 20 & 117 & 112 & 27 & 0 & 0 \\
\hline Juin $\ldots \ldots$ & 820 & 2 & 250 & 83 & 455 & 30 & 0 & 0 \\
\hline Juillet ...... & 495 & 50 & 55 & 225 & 154 & 11 & 0 & 0 \\
\hline
\end{tabular}

Mais l'influence saisonnière devient manifeste si l'on considère la rapidité de développement. En saison chaude, l'incubation ne dure pas plus d'une semaine. En hiver, le temps nécessaire à l'éclosion des œufs atteint et dépasse un mois. D'autre part, le tableau VI montre que les bulins éclos en novembre à Q. Seyed (gîte $n^{\circ} 13$ ) ont mis cinq à six mois pour atteindre la taille de 6-7 mm., correspondant à la maturité sexuelle. Trois mois ont suffi aux bulins éclos en mai pour atteindre le même résultat. 


\section{Facteurs limitants.}

Le paragraphe précédent est presque entièrement basé sur l'étude de deux gîtes seulement. C'est que, seuls, ces deux gîtes ont présenté, pendant une période suffisamment longue, des conditions favorables au développement des bulins. Cette éventualité a été rarement observée dans les 10 gîtes qui avaient été choisis pour étude longitudinale. Le plus souvent, l'un ou l'autre de deux facteurs brutalement limitants ont joué, masquant le rôle éventuel de tout autre. Ces deux facteurs sont l'asséchement et le ruissellement.

\section{Asséchement.}

Sur 10 gîtes suivis, 8 ont été soumis en cours d'année à des asséchements complets plus ou moins prolongés.

L'asséchement a été complet pour :

$\mathrm{N}^{\circ} 1$ (Ambarieh) : pendant un mois, en octobre 1960, deux mois et demi en février-mars-avril 1961.

$\mathrm{N}^{\circ} 8 a$ (Bonvar Hoceïne): pendant dix jours en janvier 1961; pendant une semaine en juin, et une autre semaine en juillet 1961 .

$\mathrm{N}^{\circ} 10$ (Nahr e Bahman) : pendant cinq jours en octobre 1960 ; pendant quatre jours en février 1961.

$\mathrm{N}^{\circ} 13$ (Q. Seyed) : pendant un mois en septembre-octobre 1960.

$\mathrm{N}^{\circ} 15$ (Qalvand) : pendant deux mois en septembre-octobre-novembre 1960 ; pendant deux mois en mai-juin 1961.

$\mathrm{N}^{\circ} 20 e$ (Biatyan): pendant un mois en mars-avril 1961.

$\mathrm{N}^{\circ} 24 e$ (Shahabad) : pendant un mois en février-mars 1960 ; pendant un mois en mai-juin 1960 ; pendant quatre jours en mars 1961, et un mois en juin-juillet 1961.

$\mathrm{N}^{\circ} 27 a$ (Chagha Sorkh) : pendant un mois en octobre-novembre 1960 ; pendant huit jours en février 1960 ; pendant un mois en mars-avril 1961, dix jours en juin et une semaine en juillet 1961 .

Il existe trois périodes pour les asséchements. Les deux premières se situent respectivement en mai-juin et en séptèmbre-octobre. A ces époques, l'eau est détournée des zones de culture continue (jardins, maraîchages), dont le drainage alimente les gîtes, pour être utilisée au maximum au profit d'autres cultures (blé, fèves, sésame). Les asséchements à ces deux époques ont réduit, toujours dramatiquement, les populations de bulins. Le gîte $\mathrm{n}^{\circ} 15$ (Qalvand) ne s'est repeuplé appréciablement qu'en février 1961, après sa mise à sec en septembre 1960 et sa remise en eau én novembre 1960. La troisième période d'asséchement se situe en hiver, l'irrigation n'étant pas pratiquée en saison pluvieuse, et les canaux étant mis à sec pour réparation. La mortalité des bulins est alors plus faible, les asséchements complets entre les périodes de 
précipitation étant relativement brefs, la température plus basse, et l'humidité atmosphérique plus forte.

\section{Résistance des bulins au desséchement.}

Par prélèvements de boue plus ou moins séchée dans le fond de gîtes connus pour avoir hébergé récemment des bulins vivants, nous avons essayé de nous rendre compte de la résistance des bulins au desséchement. Sur 50 prélèvements de boue (5), nous n'avons trouvé de bulins vivants que sept fois. Il s'agissait chaque fois de boue encore humide, et la durée de l'asséchement complet était toujours inférieure à 20 jours. En revanche, le nombre des coquilles desséchées était très important, en moyenne $12 \mathrm{par}^{\mathrm{dm}} \mathrm{de}^{3}$ boue, et jusqu'à 79 dans un échantillon (gîte $\mathrm{n}^{\circ} 24 a$, Shahabad). Le temps depuis lequel les bulins sont morts étant difficile à apprécier, on ne peut tirer de l'abondance des tests des déductions sur la mortalité due aux asséchements.

Cette faible durée de survivance dans la boue desséchée s'accorde mal avec un fait souvent constaté : le repeuplement des gîtes après de longs asséchements. Aussi nous avons voulu étudier le phénomène du desséchement dans des conditions expérimentales. Dans un terrain attenant à la station de Dizful, nous avons fait creuser de petites mares artificielles de $2 \mathrm{~m}$. de diamètre, profondes de 40 à $50 \mathrm{~cm}$., à fond d'argile. Dans la moitié de chacune d'elles, a été transplantée la végétation macroscopique couramment rencontrée dans les gîtes naturels, en particulier Typha, Juncus, Scirpus, Polygonum, Characea. Dans deux de ces mares ont été mis 500 bulins de 6 à $10 \mathrm{~mm}$., provenant de l'élevage. Au bout d'un mois (15 juin 1961), l'une des mares a été asséchée. Un mois après, le 15 juillet, la terre du fond de la mare a été entièrement lavée. 435 tests ont été retrouvés, dont 108 seulement dans la terre libre de racines et 323 entre les racines de la végétation, Typha en particulier. Entre ces racines ont également été trouvés sept bulins vivants. De cette expérience, que nous n'avons malheureusement pu ni répéter, ni étendre, il semble résulter que :

- lors des desséchements, les bulins sont susceptibles de s'enfoncer profondément le long des racines de certains végétaux aquatiques ;

- la survie au desséchement du gîte est meilleure pour les mollusques ayant ainsi pénétré entre les racines.

De toute façon, la mortalité lors des asséchements doit être considérable. Aux effets de la dessiccation s'ajoute l'activité prédatrice des oiseaux. Après un asséchement complet à l'abri de ceux-ci, tel que nous avons pu le réaliser expérimentalement, de nombreux mollusques restent à la surface du sol ayant constitué le fond du gîte. Dans les conditions naturelles, au contraire, alors

(5) Chaque échantillon mesurait $20 \times 20 \mathrm{~cm}$. horizontalement, et $10 \mathrm{~cm}$. verticalement (cf. p. 12). 
même que la boue est riche en tests, on ne trouve que peu ou pas de bulins sur la surface; celle-ci montre en revanche les traces d'un véritable « piétinement » par des petits Charadriiformes.

\section{Ruissellement.}

Sur les dix gîtes étudiés, quatre $\left(\mathrm{n}^{\circ *} 13,15,24\right.$ et 27 a) n'ont pas été sérieusement affectés par le ruissellement consécutif aux grandes pluies de décembre 1960 et janvier 1961 . Tous les autres se sont trouvés sur le passage de courants qui ont entraîné la plus grande partie des bulins. Le gîte $\mathrm{n}^{\circ} 31$ (Ab Koweit), résurgence dans le creux d'un talweg, a été entièrement bouleversé par le torrent d'eau, de boue et de galets qui l'a traversé en novembre. Aucun bulin n'y a plus été trouvé jusqu'en mars. La densité est restée extrêmement faible jusqu'en juillet. Il se peut que de telles inondations aient été la cause de la destruction des bulins dans les gîtes, très semblables à $\mathrm{Ab}$ Koweit, de $\mathrm{Ab}$ Kanar et Sar Bisheh ( $n^{\circ *} 25$ et 26) où aucun bulin vivant n'a pu être trouvé pendant plus d'un an, alors que des tests subfossiles témoignent d'un peuplement ancien (6).

Un cas assez particulier est celui du gite $\mathrm{n}^{\circ} 10$ (Nahr-e-Bahman, cf. p. 258). Servant de drain, il a, au moment des pluies de décembre et janvier, coulé à pleins bords à une vitesse supérieure à $3 \mathrm{~m}$. par seconde. Aucun bulin n'a pu y être retrouvé jusqu'en avril 1961, alors que la densité, en certains points de son trajet, avait atteint en août 1960 près de 1.000 bulins au $\mathrm{m}^{2}$ et que, sans pourtant réatteindre jamais cette densité, les populations de bulins s'étaient reconstituées rapidement après une brusque réduction, en fin octobre 1960 . La destruction hivernale avait peut-être été complète, mais le repeuplement a pu se faire à partir des drains de cultures maraîchères, au Nord de la partie amont de ce canal.

\section{Vicissitudes de l'évolution des populations de bulins.}

En somme, les rythmes saisonniers rendent mal compte de l'évolution des populations de bulins dans les gîtes étudiés. Plus exactement, au cours de nos observations, les vicissitudes imposées aux gîtes par les caprices des précipitations et des irrigations ont brouillé les rythmes saisonniers au point de les effacer. La pluviométrie, l'agriculture et, partant, l'irrigation, étant soumises à des variations saisonnières globalement régulières, on pourrait peut-être constater une évolution des populations de bulins rythmée par les saisons, si l'on pouvait faire une étude statistique de l'ensemble des populations de bulins du secteur. Mais, de l'étude d'un petit nombre de stations, telle que nous l'avons faite, se dégagent surtout deux notions:

Les fluctuations des populations de bulins au cours de l'année diffèrent

(6) Le premier de ces gîtes s'est repeuplé au printemps 1961 (voir p. 21). 
énormément de gîte à gîte. Les différents gîtes sont inégalement exposés aux effets du ruissellement. La quantité, comme les moments d'utilisation de l'eau d'irrigation, varient selon la nature des cultures localement pratiquées (maraîchage, riziculture, jardins). Les conditions de drainage varient aussi en fonction de conditions locales (perméabilité des terrains, caractère hâtif ou tardif des cultures, etc.).

Dans un gîte donné, les fluctuations des populations de bulins au cours d'une année ne reproduisent pas les fluctuations de l'année précédente. Les différences de climat d'une année à l'autre, mais surtout les assolements, contribuent à rendre les courbes d'évolution de deux années consécutives non comparables entre elles.

En définitive, si l'on considère les gîtes individuellement, il n'y a pas de rythme régulier dans l'évolution des populations de bulins. Cette évolution est une succession de périodes de croissance rapide, interrompue brusquement par des réductions brutales (assèchement, * lavages ») se succédant irrégulièrement dans le temps. Parfois, la réduction va jusqu'à l'extinction. Entre deux « réductions », la croissance des populations est plus active en automne ou au printemps qu'en plein été ou surtout en hiver; l'influence de la saison sur l'histoire du gîte s'arrête là.

Mais les grands « lavages » des gîtes n'ont pas pour seul effet la réduction ou la destruction des populations de mollusques. Chaque année, et plusieurs fois l'an, le ruissellement dû aux pluies et les inondations liées aux excès d'irrigation déterminent une vaste * redistribution 》 des bulins. Les mollusques entraînés réensemencent de nouveaux gîtes, tout aussi précaires que les premiers.

\section{Transport des bulins par agents animés.}

Le réensemencement par le ruissellement ne rend pas compte de la création de nouveaux gîtes - ou de la repopulation d'anciens gîtes - en amont de tout gîte connu (7), fait que nous avons observé à Bonvar Hoceïne (gîte $n^{\circ} 8$ ) et à $\mathrm{Ab}$ Kanar (gîte $\mathrm{n}^{\circ} 25$ ). Cette repopulation de stations restées vides pendant plus d'un an semble indiquer l'intervention d'un agent animé dans le transport des bulins. Ni dans l'un, ni dans l'autre des deux cas cités, nous n'avons pu saisir cet agent. L'intérêt de la question étant évident, sur le plan épidémiologique comme sur le plan prophylactique, nous consignons ici les observations faites dans d'autres parties du secteur sur le transport des bulins par des agents animés.

L'homme semble être un agent important. Ayant essayé de recréer à la station de Dizful des gîtes artificiels analogues aux mares entourant certains

(7) Nous avons trouvé deux gîtes au Nord du secteur, hors de celui-ci : Bidrobeh, à $40 \mathrm{~km}$ au Nord d'Andimeshk, sur la rive gauche du Bala Rud; Mal Berendji à $10 \mathrm{~km}$. au Nord-Est de Sar Bisheh. Ni l'un, ni l'autre, ne sont dans les bassins hydrographiques d'Ab Kanar ou de Bonvar Hoceïne. 
villages, nous avions décidé de les garnir de plantes aquatiques. Croyant bien faire, nos prospecteurs ont, en notre absence, garni deux de ces mares avec des plantes prélevées dans des gîtes à bulins. Ces mares se sont trouvées rapidement peuplées en limnées et en bulins ramenés avec la végétation. Il est très possible que les gîtes de Q. Seyed $\left(n^{\circ} 13\right)$, de Shahabad $\left(n^{\circ} 24\right)$ et de Chagha Sorkh $\left(\mathrm{n}^{\circ} 27\right)$ aient été peuplés en mollusques de la même façon, lorsque les villageois y ont transplanté des Typha.

Les animaux sauvages ou domestiques peuvent-ils transporter aussi des bulins ? Le rôle vecteur des oiseaux a été proposé. Nous n'avons malheureusement pu faire sur ce point aucune observation positive. En revanche, nous attirons l'attention sur deux animaux qui n'avaient pas encore été incriminés, à notre connaissance.

Ayant trouvé une limnée fixée sur la carapace d'une tortue d'eau (Clemmys sp. ?), nous avons essayé de rechercher les mollusques sur ces tortues, qui sont nombreuses dans les petits cours d'eau et se rencontrent aussi, quoique plus rarement, dans les mares stagnantes. Malheureusement, ces animaux sont difficiles à surprendre. Les seules que nous ayons réussi à capturer ne portaient aucun mollusque. Mais la façon dont, en fuyant, elles s'étaient frayé un chemin dans la végétation aquatique suffisait à expliquer cette absence. Passant une ficelle longue de $50 \mathrm{~cm}$. dans un trou perforé au bord de la carapace, nous avons alors " mis au piquet " deux Clemmys dans le gîte de Q. Seyed. Au cours des visites ultérieures, nous avons toujours trouvé des bulins sur la carapace de nos prisonnières. Nous n'y avons jamais observé de pontes.

Nous nous étions demandé par ailleurs si les bulins n'étaient pas susceptibles de s'attacher au cuir pauvre en poils des buffles, au cours des longues stations que ces animaux font, immergés presque complètement, dans l'eau de certains gîtes. Nous avons examiné une vingtaine de buffles dérangés de leur baignade dans le gîte $\mathrm{n}^{\circ} 35 b$, à Shamoun. Nous n'avons trouvé aucun bulin sur le cuir de ces animaux. En revanche, nous en avons trouvé un dans la boue retenue entre les deux sabots d'une de ces bêtes. Ceci suggère que, non seulement les buffles, mais aussi d'autres animaux paissant dans des gites à bulins peuvent, occasionnellement, transporter ces derniers.

\section{Biologie des bulins et bilharziose}

\section{A. Contacts homme-mollusques.}

Les contacts « homme-mollusques 》 sont manifestes dans les petites mares creusées à proximité des villages (cf. p. 246). Les baignades sont plutôt rares dans ces mares, mais il existe d'autres occasions de contact et d'infection. Les hommes viennent y prendre et manipuler la boue argileuse. Les femmes vien- 
nent occasionnellement y laver le linge et les ustensiles ménagers ; dans certaines circonstances, elles y prélèvent même l'eau de boisson. Les enfants jouent volontiers dans ces mares, où l'eau est d'accès plus proche, plus facile et moins dangereux qu'il n'est ailleurs. Les mares sont évidemment les lieux de la contamination humaine pour de nombreux villages: Shahabad, Sardarabad, Salarabad, Shamoun, Deylam Sofla, etc. (stations $\mathrm{n}^{\circ \mathrm{s}} 24,33,34,35,36$, etc.). Cependant, la présence d'une mare à bulins à proximité immédiate d'un village ne signifie pas nécessairement une prévalence élevée de l'infection humaine. Des villages comme Q. Seyed ou Shalghehi Olia, ayant à leur porte des mares abondamment peuplées de bulins (gîtes $n^{\circ *} 13$ et 29) présentent des indices globaux d'infection assez bas (respectivement 11 et $8 \%$ ). Dans le village de Chagha Sorkh, au centre duquel se trouve une mare à bulins (gîte $\mathrm{n}^{\circ} 27 a$ ), $6 \%$ seulement des habitants sont infectés. Nous envisagerons dans les chapitres suivants ( $B$ et $C$ ) des hypothèses qui expliquent peut-être ces faits paradoxaux.

Les petites sources qui forment, dans la partie Nord-Est du secteur, quelques gîtes à bulins $\left(\mathrm{n}^{\circ \pi} 25,26,31\right)$ jouent un rôle épidémiologique en tout point comparable à celui des mares. Les contacts homme-mollusques y sont plus étroits encore qu'ils ne le sont dans les mares, ces sources représentant généralement les seuls points d'eau utilisables au sein d'étendues de terres arides. Il semble que ce soit à la suite d'une épidémie de bilharziose que le petit village d'Ab Koweit, construit autour du gîte $\mathrm{n}^{\circ} 31$, ait été abandonné il y a cinq ans par ses habitants.

En plus des contacts \& professionnels » à l'occasion des travaux agricoles, les canaux sont, de préférence à toute autre collection d'eau, le lieu des baignades et, lorsqu'ils sont proches des villages, du lavage du linge et des ustensiles ménagers. Ils sont rarement un lieu de reproduction pour les bulins. Ils en hébergent pourtant, occasionnellement, et sont alors, au même titre que les mares, le lieu de contacts homme-mollusques directs. Ainsi les gîtes $n^{\circ *} 114$ et 115 , dans les branches tertiaires du canal Nahr e Gumat, sont certainement responsables de l'infection des villages de Seyed Mohcine et Seyed Hassane. Mais les canaux interviennent aussi d'une façon plus indirecte dans l'épidémiologie de la bilharziose. Les canaux des réseaux d'aval récoltent très souvent les eaux de drainage des réseaux d'amont (cf. p. 238). Lorsque des gîtes à bulins existent dans les eaux de drainage du réseau supérieur, les canaux du réseau inférieur peuvent véhiculer assez loin les cercaires émises par ces bulins. Les canaux d'irrigation peuvent ainsi, sans héberger de bulins, faire trait d'union entre mollusques et humains, jouant ainsi un rôle important dans l'infection de ces derniers (8).

(8) Une étude particulière a été faite sur le réseau du canal Nahr e Shahabad. Toute la surface couverte par ce réseau a été surveillée régulièrement de mai 1960 à mai 1961. Deux villages existent sur cette surface : celui de Shahabad, au centre du réseau; celui de $Q$. Now Sardar, $2 \mathrm{~km} 1 / 2$ en aval du premier. 
Les grands marécages naturels, les marécages dus au drainage insuffisant des dépressions naturelles, ou ceux créés par des levées de terre artificielles, constituent généralement des pâturages d'été. Les enfants commis à la garde des bêtes sont dans des conditions parfaites pour infecter les bulins vivant dans ces marécages et s'infecter en retour.

Les rizières, dans les conditions actuelles, sont rarement des gites à bulins, et ceux-ci y sont toujours peu nombreux. Le désherbage des rizières n'est pas pratiqué pour le moment et la moisson est faite après assèchement. Dans ces conditions, le rôle épidémiologique des rizières doit être faible ou nul.

\section{B. Pouvoir infectant des bulins.}

Trois sortes de cercaires s'observent chez les bulins du secteur: Paramphistomum cervi, Schistosoma haematobium et Schistosoma bovis. Si les cercaires du premier Trématode sont faciles à distinguer des autres, il est à peu près impossible de séparer les cercaires des deux espèces de schistosomes sans recourir à des infections expérimentales. Faute d'animaux en nombre suffisant, nous avons dû renoncer aux identifications systématiques d'espèce. Lorsque nous parlons de bulins infectés, il faut entendre « infectés par des schistosomes de l'une ou l'autre espèce, ou des deux simultanément ».

L'évolution des schistosomes chez les bulins ne semble pas avoir lieu tout au long de l'année. Aucune cercaire n'a pu être trouvée pendant les mois de janvier, février et mars. En avril 1960, nous avons pu trouver des cercaires chez les bulins du gîte $n^{\circ} 22$ (Farashabad). En avril 1961, nous n'avons pu mettre en évidence aucune cercaire. En mai 1961, aucun des bulins récoltés dans les dix gîtes régulièrement suivis n'était parasité ; mais nous avons pu trouver des cercaires de schistosomes chez un bulin (sur 99 examinés) du gîte $\mathrm{n}^{\circ} 35 a$, à Shamoun (9). Les mois de septembre, octobre et novembre semblent ceux où le pouvoir infectant des bulins est au maximum.

Les mollusques infectés sont rarement nombreux dans un gîte donné. Les indices d'infection les plus élevés ont été observés en octobre chez les bulins du canal Nahr e Bahman : $3 / 33$ dans le gîte $\mathrm{n}^{\circ} 10 ; 7 / 492$ dans le gîte $\mathrm{n}^{\circ} 11 a$ (à noter que l'infection touchait électivement les bulins de taille supérieure à

Aucun bulin vivant n'a été trouvé au-delà d'un rayon de $600 \mathrm{~m}$. autour de Shahabad. Dans les murs de $Q$. Now Sardar, et sur les berges d'un canal entre les deux villages, ont été trouvés seulement des tests subfossiles. Les indices d'infection humaine au printemps 1961 étaient de $32 \%$ pour Shahabad et de $39 \%$ pour $Q$. Now Sardar. La répartition de l'infection par groupes d'âges montre que des contaminations ont eu lieu à $Q$. Now Sardar au cours de l'automne 1960. Il faut donc admettre que de jeunes enfants de Q. Now Sardar ont été infectés par des cercaires provenant des bulins de Shahabad.

De même, après avoir cherché vainement pendant des mois le gîte responsable de l'infection bilharzienne à Boneh Lussi, petit village à l'Ouest du secteur, à la terminaison d'une branche du réseau Harmushi, nous avons dû arriver à la conclusion que les cercaires infectantes provenaient de gîtes situés à $2 \mathrm{~km}$. au moins en amont (Gites $n^{\circ} 38,39$, ou 65 ).

(9) L'examen de 130 bulins du même gîte avait été négatif en avril. 
$8 \mathrm{~mm}$ : : 3/7 dans le gîte $\mathrm{n}^{\circ} 10 ; 6 / 92$ dans le gîte $\mathrm{n}^{\circ} 11$ a). A ces deux exceptions près, nous n'avons jamais observé d'indice supérieur à $1 \%$. Sans doute n'avons-nous pas pu surprendre, dans d'autres gîtes, le bref moment favorable que nous avons eu la chance de saisir au Nahr e Bahman.

$\mathrm{Si}$, en effet, la densité des mollusques infectés est forte, ce n'est jamais pendant une longue période. Les fréquentes et brusques réductions des populations de bulins expliquent, dans un certain nombre de cas, cette rareté des mollusques parasités. La rareté des bulins porteurs de cercaires est tout aussi remarquable, mais moins compréhensible, dans les cas où les bulins se développent sans accident pendant plusieurs mois. Nous avons, de mai à juillet 1961, écrasé plusieurs centaines de bulins provenant de $Q$. Seyed (gîte $n^{\circ} 13$ ) sans en trouver un seul parasité. Ce gîte n'avait pas subi d'assèchement complet depuis plusieurs mois. On peut se demander si, dans les gîtes surpeuplés en bulins et où la mortalité de ceux-ci est élevée (voir, tableau VII, l'évolution de la structure des populations de bulins de Q. Seyed), les mollusques infectés ne supportent pas particulièrement mal la concurrence vitale et ne présentent pas une mortalité plus élevée que les mollusques sains.

De cette faible densité des mollusques infectés résulte la rareté des réinfections humaines subintrantes. Cette rareté explique peut-être la bénignité clinique des infections bilharziennes au Khouzistan, bénignité qui coexiste, et contraste, avec des prévalences élevées.

Les variations d'une année à l'autre dans l'incidence de la maladie parmi la population d'un village donné, l'apparition de nouveaux foyers et la disparition de certains autres s'expliquent par la fréquence des destructions totales de la population des mollusques d'une station (assèchements, grands « lavages »), d'une part, et par les possibilités de colonisation offertes aux bulins à l'occasion de ces mêmes grands « lavages », d'autre part.

\section{Corrélation entre distribution des bulins et distribu- tion de la bilharziose.}

La comparaison des cartes 3 et 4 , montrant la distribution de $B$. truncatus dans le secteur d'étude, avec la carte 5 (10) schématisant nos connaissances sur la prévalence de la bilharziose dans le même secteur, est assez surprenante. L'infection bilharzienne est supérieure à $20 \%$ dans les $2 / 3$ Sud-Est du secteur d'étude. La prévalence est inférieure à $10 \%$, et souvent nulle, dans le quart Nord-Ouest du secteur. Cependant, il n'existe pas de différence importante

(10) Cette carte a été établie, partie avec les données recueillies antérieurement (1958-59), par l'Institut de Parasitologie de Téhéran, partie avec les données de la minutieuse enquête menée dans la zone-pilote d'irrigations et dans la zóne au Sud de Shush par le $\mathrm{D}^{\mathrm{r}}$ Gremliza en 1959-60-61 (cette enquête recoupe parfaitement la précédente), partie enfin avec les données recueillies par nousmêmes en 1960-61. 
entre la densité des bulins dans la zone fortement infectée et dans la zone relativement indemne. La densité des stations de bulins est de 6,9 au $\mathrm{km}^{2}$ dans la première et de $5 \mathrm{au} \mathrm{km}^{2}$ dans la seconde, la différence n'étant toutefois pas significative, statistiquement parlant.

Nous avons cherché si le type des gîtes, leur proximité plus ou moins grande des villages, le nombre de gîtes par station, les densités de peuplement des gîtes par les bulins pouvaient expliquer cette division du secteur en deux zones, l'une très infectée de bilharziose, l'autre pratiquement indemne. Il est à noter que la présence de bulins dans les canaux d'irrigation est presque un privilège de la zone infectée. Mais ce type de gîtes ne s'observe pas sur toute la zone infectée. Il domine le long de la bordure Sud-Ouest de celle-ci; il est absent au centre et au Nord. L'explication n'est donc pas là. La proximité entre gîte et village ne paraît pas jouer non plus. Nous avons cité plus haut les cas démonstratifs de Q. Seyed, Shalghehi Olia, Chagha Sorkh, où une extrême proximité du village et du gîte coïncident avec des indices d'infection faibles. Le nombre de gîtes par station ne dépasse deux qu'en quatre points. Trois de ceux-ci se trouvent dans la zone infectée, en particulier les deux plus riches: Biatyan ( $n^{\circ} 20,5$ gîtes) et Shahabad ( $n^{\circ} 24,7$ gîtes). Mais tant de villages sont fortement infectés alors qu'un seul gîte se trouve à proximité - ou même sans qu'aucun gîte actuel ait pu être trouvé à proximité - que nous hésitons à voir là l'explication cherchée. Quant à la densité de peuplement des gîtes, outre le cas de Q. Seyed, cité au paragraphe précédent, notons qu'à Q. Now Shamsabad, traversé par le Nahr e Bahman en aval des gîtes les plus riches connus dans ce canal, la prévalence de la bilharziose est inférieure à $5 \%$.

Cherchant ailleurs une explication à cette division du secteur en deux zones si inégalement infectées, on remarque qu'il existe une corrélation assez étroite entre l'origine ethnique des populations et la prévalence de la bilharziose. Les populations arabes sont fortement infectées, ainsi que les populations Bakhtiari, au sein desquelles de nombreux petits groupes d'Arabes se sont infiltrés. Les populations Lur et Dizfuli sont très peu touchées. Cette corrélation est peutêtre fortuite. Si elle était valable, plusieurs hypothèses pourraient l'expliquer : 1) un comportement différent des villageois vis-à-vis de l'eau, fait que nous n'avons pas pu constater; 2) un brassage des différents éléments ethniques insuffisant jusqu'ici pour que la contamination des collectivités indemnes ait pu se produire.

Comme hypothèse d'explication, on pourrait penser encore à une extension relativement récente de l'œcoumène de $\boldsymbol{B}$. truncatus, que l'infection bilharzienne ne suivrait qu'avec retard. A ce sujet, remarquons qu'il nous est deux fois arrivé de trouver des tests de bulins au sommet de petites collines, en fait vestiges de constructions anciennes (tells). Ces tests provenaient probablement de la terre des murs détruits. Ces deux trouvailles ont été faites respectivement à Seyed Mohcine et à Deh i Now, les deux localités se trouvant dans le tiers 
Sud-Est du secteur d'étude, soit dans la zone où la prévalence de la bilharziose est forte.

\section{Conclusions}

\section{A. Résumé des données recueillies sur la biologie des bulins.}

Il existe une disproportion entre l'abondance et l'étendue des collections d'eau dans le secteur étudié d'une part et, d'autre part, la faible extension des colonies de Bulinus truncatus. Les stations de $B$. truncatus sont peu nombreuses, assez isolées les unes des autres; l'étendue des gîtes est généralement faible.

Les gîtes « naturels 》 (grands marécages, petites sources) sont peu nombreux. Le développement des bulins y est peu abondant. La vie des bulins, dans le secteur étudié, est étroitement liée aux activités humaines, essentiellement à l'irrigation. Contrairement à ce qui s'observe dans d'autres pays, les canaux d'irrigation eux-mêmes ne constituent pas les gites les plus nombreux ni les plus importants. Ces canaux hébergent occasionnellement des bulins et en assurent très probablement la dissémination. Mais, sauf circonstances tout à fait exceptionnelles (cas du canal Nahr e Bahman, cf. p. 15), les canaux ne sont pas un lieu de reproduction favorable pour les bulins. Les cultures irriguées, ou même submergées (riz), constituent exceptionnellement des lieux de reproduction pour les bulins.

Les gîtes de $B$. truncatus sont essentiellement créés par des défauts de drainage. Les eaux d'irrigation en excès, ou provenant de fuites et de débordements des canaux d'irrigation, constituent des gîtes lorsqu'elles stagnent faute de pouvoir atteindre des drains naturels. Les causes de cette stagnation sont :

- l'existence de dépressions naturelles;

- la retenue involontaire des eaux par des barrages artificiels : routes, voie ferrée, levées de terre de canaux actuellement en service ou d'anciens canaux abandonnés;

- la création volontaire, à proximité immédiate des villages, de mares destinées à fournir la boue argileuse, élément important de la vie sociale du pays. Cette dernière cause est fréquente et certains villages sont entourés d'une véritable ceinture de telles mares. Dans certaines d'entre elles, les villageois ont transplanté des Typha, dont les feuilles sont utilisées pour lier les gerbes de riz au moment du repiquage; cette végétation aquatique semble favoriser au maximum le développement des bulins. 
Dans tous ces gîtes artificiels, l'équilibre biologique des populations de bulins est précaire. L'activité reproductrice s'observe tout au long de l'année. Le développement des mollusques est souvent extrêmement actif et la densité de peuplement par unité de surface devient rapidement extraordinaire. Mais elle ne se maintient pas. La discontinuité dans l'irrigation entraîne des périodes de dessiccation des gîtes qui arrêtent le développement des mollusques et causent parfois l'entière destruction des bulins. Par ailleurs, les grandes pluies « lavent » beaucoup de gîtes, détruisant et emportant au loin une grande partie des mollusques.

En revanche, chaque grande pluie est une occasion de « redistribution » des bulins sur de vastes surfaces. Etant donné les nombreuses anastomoses existant entre les divers réseaux d'irrigation, les bulins entraînés à partir d'un gîte donné peuvent s'arrêter et créer des colonies nouvelles en des points imprévisibles, répartis sur une vaste surface.

Les deux types de collections d'eau les plus favorables aux contacts hommemollusques et à la transmission de la biaharziose sont les petites mares creusées à proximité immédiate des villages et les canaux d'irrigation. Les mares sont à la fois gîtes à bulins et lieux de la contamination humaine. Les canaux peuvent être lieux de contamination humaine sans être gîtes à bulins. A cause des anastomoses complexes entre drains et canaux, ces derniers peuvent véhiculer les cercaires émises par des bulins vivant dans les premiers.

L'infection des bulins par des cercaires de schistosomes ne s'observe pas tout au long de l'année. La transmission de la bilharziose est pratiquement arrêtée pendant les quatre premiers mois de l'année (janvier à avril). Les taux d'infection observés chez les mollusques sont généralement remarquablement faibles, ce qui est, en partie du moins, une conséquence de la discontinuité dans l'évolution des populations de bulins. Cette discontinuité est vraisemblablement la raison essentielle de la bénignité relative actuelle de la bilharziose urinaire dans le secteur étudié.

Le passage de l'irrigation discontinue actuellement pratiquée, à l'irrigation pérenne, doit favoriser considérablement le développement des bulins et de la bilharziose, si des mesures palliatives ne sont pas prises. La dessiccation estivale meurtrière pour les bulins sera en effet évitée à beaucoup plus de gîtes qu'elle ne l'est dans les conditions actuelles. La stabilité plus grande des populations de mollusques donnera au cycle évolutif des schistosomes de meilleures chances de s'établir et de se maintenir. On observera non seulement une élévation du nombre des individus infectés, mais aussi, probablement, une gravité moyenne plus grande des manifestations cliniques de l'infection. 


\section{B. Déductions quant à la prévention de la bilharziose.}

Sans qu'on puisse l'affirmer facile, la lutte contre les bulins est beaucoup plus prometteuse au Khouzistan qu'elle ne l'est dans d'autres pays. Ceci tient :

— à la faible surface des gîtes où a lieu une reproduction active ;

- au fait que ces gîtes se trouvent en eau stagnante le plus généralement ;

- au fait que ni les canaux d'irrigation, ni les cultures irriguées ne sont des lieux de reproduction importants pour $B$. truncatus.

Dans ces conditions, la lutte contre les bulins aura des chances d'efficacité très supérieures à celles de la lutte contre le réservoir humain de parasites et sera moins onéreuse que cette dernière.

La méthode de lutte idéale paraît être la suppression complète des gîtes de reproduction. On devra envisager dans ce but trois ordres de mesures:

1) Une régulation des débits en tête de canal, qui réduirait les excès d'eau en aval. Cette mesure aurait en même temps des avantages pour l'agriculture et réduirait la dégradation des routes.

2) Le drainage des dépressions naturelles, l'ouverture de brèches ou le percement de tunnels dans, ou sous, les barrages (routes, voie ferrée, levées de terre diverses) entravant l'écoulement des eaux vers les drains naturels. Cette mesure lutterait en même temps contre la remontée en surface des sels du sous-sol et aurait des avantages agricoles. Nous ne pouvons pas dire jusqu'à quel point la réduction des pâturages d'été que représentera la suppression des gîtes à bulins devra être compensée par des cultures fourragères.

3) La suppression des mares entretenues à proximité immédiate des villages. Celle-ci soulèvera certainement des difficultés. Elle sera possible seulement si le ravitaillement en eau des villages est assuré tout au long de l'année, soit par un débit des canaux maintenu en hiver, soit par la création de puits ou de citernes. A défaut de la suppression complète, on peut envisager l'interdiction aux villageois de conserver plus d'un mois de l'eau dans une même mare. La constatation, dans une mare en eau, d'une végétation aquatique ou d'un abondant développement d'algues vertes serait le test d'un séjour trop prolongé de l'eau et entraînerait des sanctions à déterminer.

La création en dérivation sur les canaux proches des villages d'abreuvoirs pour les bêtes, de lavoirs sommaires, voire de petits bassins de natation où un produit cercaricide assurerait la sécurité de la baignade, n'est pas une nécessité au point de vue de la prévention de la bilharziose ; il en est de même de la création de latrines. Toutefois, ces créations, en même temps qu'une compensation à la contrainte exercée, constitueraient un pas vers l'équipement sanitaire des villages et donc vers l'avenir. 
Pendant une période transitoire aussi courte que possible, les molluscocides pourront être utilisés pour neutraliser les gîtes non détruits. Les concentrations employées devront être fortes, et les applications fréquentes, car la dureté et l'alcalinité des eaux d'une part, la forte insolation d'autre part, l'abondante végétation des gîtes enfin inactiveront rapidement l'effet des molluscocides. En revanche, la faible surface des gîtes permet d'envisager de fortes concentrations en produits actifs sans élever déraisonnablement le prix de la lutte.

Quelques réunions d'information touchant les propriétaires et une éducation sanitaire donnée aux moniteurs agricoles formés par l'Ecole de Mollah Sani compléteraient heureusement cet ensemble de mesures.

$\mathrm{Si}$ ce tableau incite à l'optimisme, une réserve doit pourtant être faite. Une régulation des débits d'eau en tête des canaux est proposée ci-dessus. Par ailleurs, certains aménagements de ces canaux (remembrements, rectifications, protection contre les eaux de ruissellement, revêtements, etc.) seront peut-être jugés nécessaires pour des raisons de technique agricole ou hydraulique, pour l'économie de l'eau ou la récupération de terres. Il n'est pas exclu que ces améliorations, ramenant le réseau vétuste du Khouzistan aux conditions des réseaux plus modernes d'autres pays, ne rendent ce réseau habitable pour les bulins, comme le sont précisément les réseaux plus modernes.

Il est impossible d'estimer la gravité de ce danger, dans l'ignorance où nous sommes des raisons pour lesquelles les canaux du Khouzistan sont généralement impropres au développement des bulins. La prudence commande de ne pas faire de modifications générales des canaux d'irrigation sans avoir au préalable étudié, dans des projets pilotes à petite échelle, les éventuelles modifications écologiques entraînées par les améliorations projetées.

De même, il faudra suivre avec attention, sur des projets pilotes, les résultats de toute modification de technique jugée nécessaire pour l'amélioration de toute culture abondamment irriguée, riziculture particulièrement. Une de ces modifications peut, imprévisiblement, offrir aux bulins les conditions écologiques favorables qui font actuellement défaut.

La zone-pilote d'irrigations est le lieu désigné pour de telles expériences. Les épidémiologistes auront à suivre de très près l'évolution de cette zone pilote, pour savoir dans quelle mesure encourager la généralisation de certaines pratiques nouvelles ou, au contraire, s'opposer à leur extension. 